\title{
Paracardial fat remodeling affects systemic metabolism through alcohol dehydrogenase 1
}

\author{
Jennifer M. Petrosino, Jacob Z. Longenecker, ${ }^{1}$ Srinivasagan Ramkumar, ${ }^{2}$ Xianyao Xu, ${ }^{3}$ Lisa E. Dorn, ${ }^{1}$ Anna Bratasz, ${ }^{4}$ Lianbo Yu, ${ }^{5}$ \\ Santosh Maurya, ${ }^{1}$ Vladimir Tolstikov, ${ }^{6}$ Valerie Bussberg, ${ }^{6}$ Paul M.L. Janssen, ${ }^{1}$ Muthu Periasamy, ${ }^{1,7}$ Michael A. Kiebish, ${ }^{6}$ \\ Gregg Duester, ${ }^{8}$ Johannes von Lintig, ${ }^{2}$ Ouliana Ziouzenkova, ${ }^{9}$ and Federica Accornero ${ }^{1}$

\begin{abstract}
'Department of Physiology and Cell Biology, Dorothy M. Davis Heart and Lung Research Institute, The Ohio State University, Columbus, Ohio, USA. ${ }^{2}$ Department of Pharmacology, Case Western Reserve University, Cleveland, Ohio, USA. ${ }^{3}$ Department of Biomedical Engineering, Dorothy M. Davis Heart and Lung Research Institute, ${ }^{4}$ Small Animal Imaging Core, ${ }^{5}$ Center for Biostatistics, Department of Biomedical Informatics, The Ohio State University, Columbus, Ohio, USA. ${ }^{6}$ BERG, Precision Medicine Department, Framingham, Massachusetts, USA. 'Department of Internal Medicine, University of Central Florida, Orlando, Florida, USA. ${ }^{8}$ Development, Aging, and Regeneration Program, Sanford Burnham Prebys Medical Discovery Institute, La Jolla, California, USA. ${ }^{9}$ Department of Human Sciences, College of Education and Human Ecology, Comprehensive Cancer Center, The Ohio State University, Columbus, Ohio, USA
\end{abstract}

\begin{abstract}
The relationship between adiposity and metabolic health is well established. However, very little is known about the fat depot, known as paracardial fat (pCF), located superior to and surrounding the heart. Here, we show that pCF remodels with aging and a high-fat diet and that the size and function of this depot are controlled by alcohol dehydrogenase 1 (ADH1), an enzyme that oxidizes retinol into retinaldehyde. Elderly individuals and individuals with obesity have low ADH1 expression in pCF, and in mice, genetic ablation of $A d h 1$ is sufficient to drive pCF accumulation, dysfunction, and global impairments in metabolic flexibility. Metabolomics analysis revealed that pCF controlled the levels of circulating metabolites affecting fatty acid biosynthesis. Also, surgical removal of the pCF depot was sufficient to rescue the impairments in cardiometabolic flexibility and fitness observed in Adh1-deficient mice. Furthermore, treatment with retinaldehyde prevented pCF remodeling in these animals. Mechanistically, we found that the ADH1/retinaldehyde pathway works by driving PCC-1 $\alpha$ nuclear translocation and promoting mitochondrial fusion and biogenesis in the pCF depot. Together, these data demonstrate that pCF is a critical regulator of cardiometabolic fitness and that retinaldehyde and its generating enzyme ADH1 act as critical regulators of adipocyte remodeling in the pCF depot.
\end{abstract}

\section{Introduction}

Adipose tissue is known as both a storage and secretory organ capable of influencing local and global metabolism (reviewed in refs. 1, 2). In particular, white adipose tissue acts as an energy reservoir that, in response to obesogenic conditions, undergoes a dynamic remodeling that causes increases in adipocyte size and number (reviewed in ref. 1). This type of maladaptive adipose remodeling is associated with changes in the organ's secretome that subsequently impairs global metabolism and metabolic flexibility (ref. 3 and reviewed in refs. 4, 5).

Preserving metabolic flexibility, the adaptability of an organism to metabolic or energetic stress, is essential for the regulation of homeostasis $(6,7)$ and key to maintaining optimal health. Metabolic flexibility is necessary for organs and cells to adjust fuel substrate selection during periods of altered energetic demands (8). Metabolic inflexibility is observed in obesity and obesity-related cardiometabolic diseases, in which reductions in mitochondrial mass drive adipocyte expansion and promote the dysfunction of

Related Commentary: https://doi.org/10.1172/JCI145969

Conflict of interest: The authors have declared that no conflict of interest exists. Copyright: () 2021, American Society for Clinical Investigation.

Submitted: June 29, 2020; Accepted: December 10, 2020; Published: February 15, 2021.

Reference information: J Clin Invest. 2021;131(4):e141799.

https://doi.org/10.1172/JCl141799. cellular and systemic energetics (refs. 9-12 and reviewed in ref. 13). Hence, with adipose tissue expansion acting as a detrimental driver of metabolic inflexibility in obesity and as a risk factor for cardiovascular disease (14-18), it is critical to understand how adipose tissue expansion and function are regulated as a means to prevent or reverse metabolic dysfunction.

In general, much is known about the role of brown, subcutaneous, and intra-abdominal visceral adipose tissues in disease pathogenesis (reviewed in ref. 19), and it is well established that fat itself is an endocrine organ $(20,21)$. However, very little is known about the specific contribution of the fat depot that surrounds the heart, its effects on cardiometabolic health, and its capabilities as an active endocrine tissue. The fat that surrounds the heart can be divided into 3 types: epicardial, pericardial, and paracardial. Epicardial adipose tissue is derived from the splanchnopleuric mesoderm and is located between the outer wall of the myocardium and the visceral layer of the pericardium. The second type, pericardial fat, is anterior to epicardial fat and therefore located between the visceral and parietal pericardium. Primitive thoracic mesenchyme-derived paracardial fat $(\mathrm{pCF})$ is the third type and is located superior to the visceral pericardium and around the blood vessels $(18,22-24)$. Accumulation of pCF tissue is observed in individuals with obesity and is considered a predictor for coronary artery events (25-27). Despite this knowledge, the lack of genetic models available to study the pCF depot has left the biology and mechanisms driving its expansion undefined. 
A
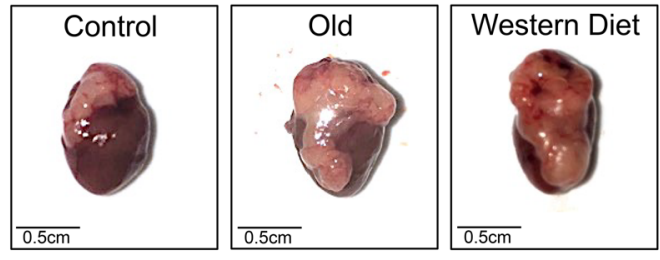

$\mathbf{E}$
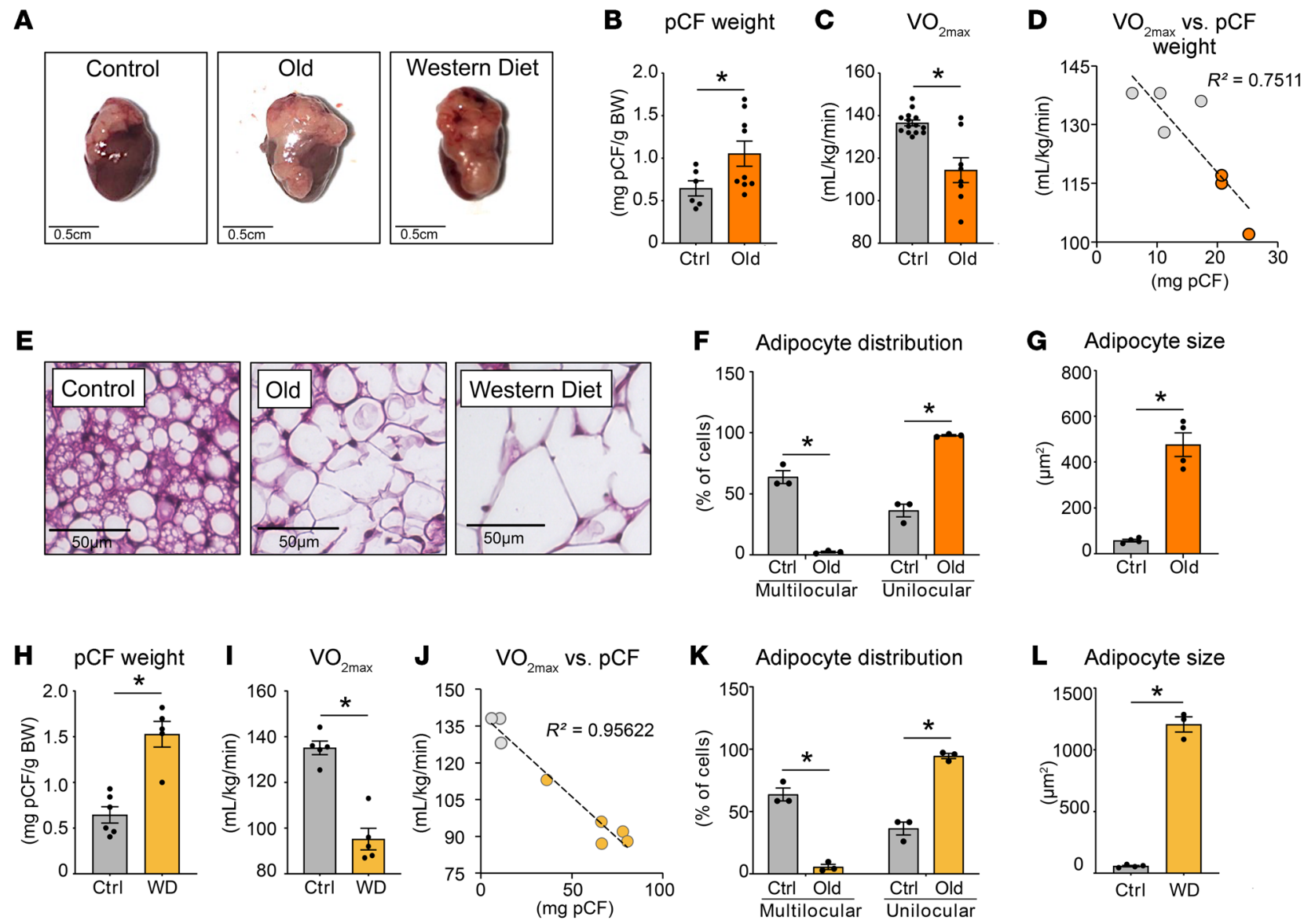

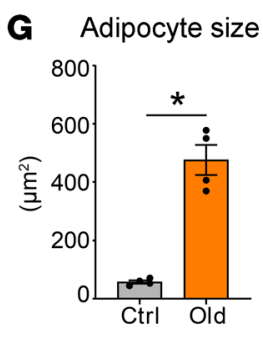

L Adipocyte size

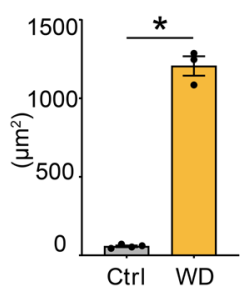

Figure 1. pCF is a unique fat depot that expands with aging and obesity. (A) Representative images of pCF on top of hearts from control diet-fed (6 months old), control diet-fed old mice (20 months old), and Western diet-fed mice (6 months old; 12 weeks on a Western diet). Scale bars: $0.5 \mathrm{~cm}$. (B) pCF weight normalized to $\mathrm{BW},(\mathbf{C})$ maximal oxygen consumption $\left(\mathrm{VO}_{2 \max }\right)$ during graded maximal exercise testing, and (D) relationship between $\mathrm{VO}_{2 \max }$ and the amount of pCF for control (Ctrl) and old animals. (E) Representative H\&E-stained images of the pCF depot from control, old, and Western diet-fed mice. Scale bars: $50 \mu \mathrm{m}$. (F) Distribution of multilocular and unilocular adipocytes and (G) unilocular adipocyte size for control and old animals. (H) pCF weight normalized to $\mathrm{BW}$, (I) $\mathrm{VO}_{2 \max }$ during graded maximal exercise testing, and (J) relationship between $\mathrm{VO}_{2 \max }$ and the amount of pCF for control diet-fed and Western diet-fed (WD) animals. (K) Distribution of multilocular and unilocular adipocytes and (L) unilocular adipocyte size for control and Western dietfed animals. For pCF weight and exercise testing, $n=5-14$ per biological animal replicate. For adipocyte quantifications, $n=75-150$ for a given biological replicate for adipocyte size and $n=150-275$ for adipocyte numbers for a given biological replicate, and $n=3-4$ biological replicates per genotype. Data are presented as the mean \pm SEM or by dot plot with linear regression. $P=0.05$, by Student's $t$ test.

Here, we have characterized the pCF depot, identified the mechanisms that drive its expansion, and determined its biological significance. Specifically, we found that the retinol pathway is critical for PCF pathophysiology. Our data highlight how alcohol dehydrogenase 1 (ADH1) and its product retinaldehyde are important regulators of pCF remodeling and critical effectors of cardiometabolic flexibility.

\section{Results}

$p C F$ remodeling correlates with cardiometabolic impairments and alterations in ADH1 expression with aging and obesity. $\mathrm{pCF}$ is a poorly characterized depot. We found that pCF accumulated with aging and following 12 weeks of a Western diet in mice (Figure $1, \mathrm{~A}, \mathrm{~B}$, and $\mathrm{H})$. Considering the proximity of $\mathrm{pCF}$ to the heart and the reliance of the heart on free fatty acids to support its metabolic needs, we tested the cardiometabolic fitness of aged and Western diet-fed mice using a graded maximal exercise test (28). This test revealed that exercise performance impairments, as shown by decreased maximal oxygen consumption $\left(\mathrm{VO}_{2 \max }\right)$, correlated with the accumulation of $\mathrm{pCF}$ with aging and Western diet feeding (Figure 1, C, D, I, and J). Histologically, we found that $\mathrm{PCF}$ was a heterogeneous depot characterized by a mixture of unilocular and multilocular adipocytes, and that with aging and Western diet feeding, pCF remodeled to acquire a phenotype that better resembled classical white adipose tissue (Figure 1E). Indeed, adipocyte distribution analysis revealed a decreased number of multilocular cells and an increased number of unilocular cells with both aging and Western diet feeding (Figure 1, F and K). Further assessment of unilocular adipocyte size showed adipocyte hypertrophy in the $\mathrm{pCF}$ of old and Western diet-fed mice (Figure 1, G and L).

The exact nature and molecular signature of $\mathrm{pCF}$ is unknown. It is also not defined how $\mathrm{PCF}$ is distinct from classical visceral fat depots such as the intra-abdominal visceral fat (iVF) one. To test how this fat depot surrounding the heart differed from iVF, we performed genome-wide transcriptome analysis comparing the mRNA profile 
A

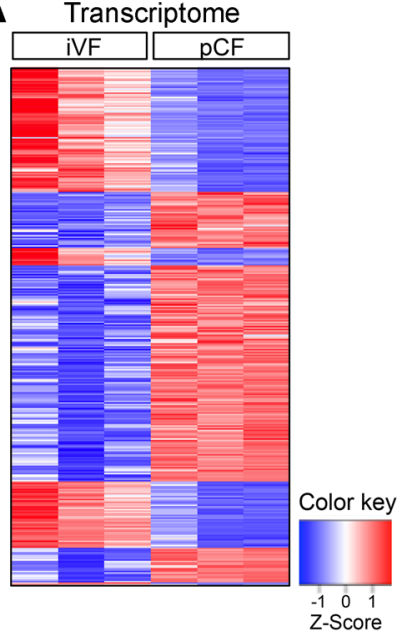

B

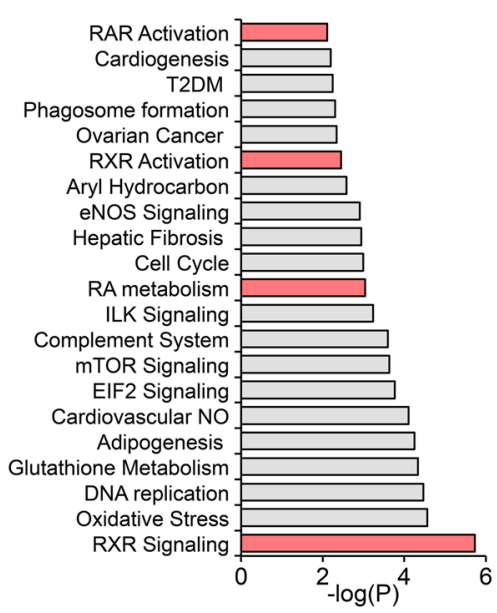

C Retinol oxidation

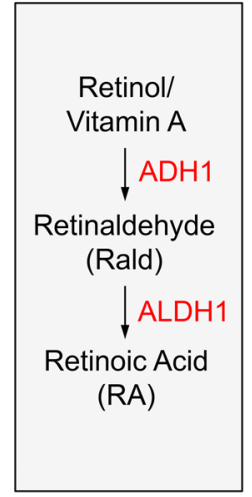

D Pericardial fat
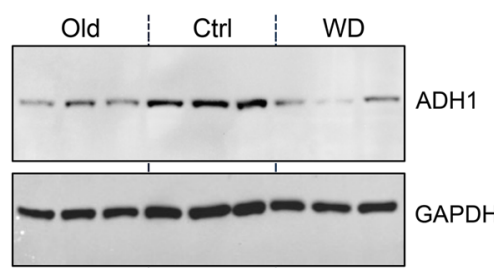
GAPDH

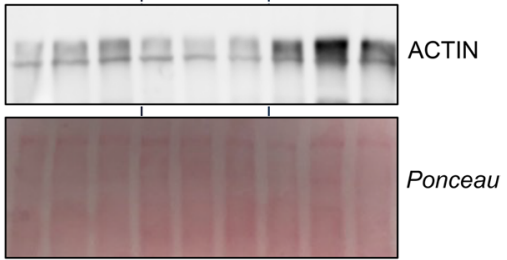

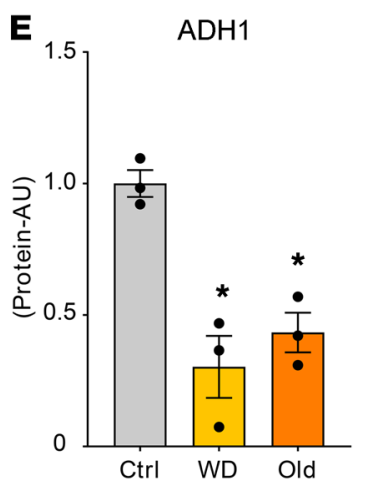
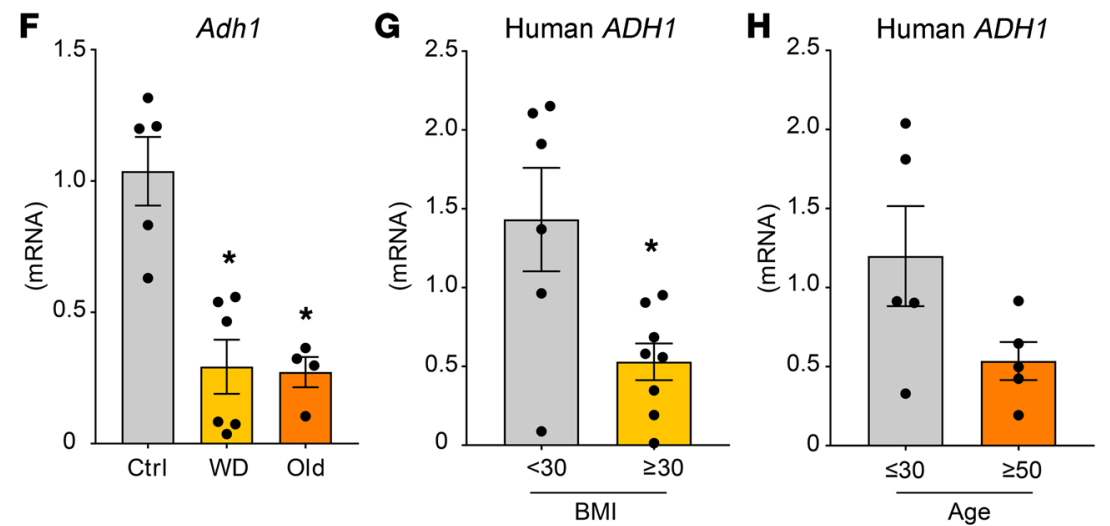

Figure 2. Importance of Adh1 expression for pCF biology. (A) Heatmap and (B) the top most significantly regulated canonical pathways determined by IPA from a list of 1023 genes that were differentially regulated between WT iVF and pCF using the Affymetrix transcriptome array. (C) Schematic of the retinol oxidation pathway. (D) Western blot and (E) quantification of ADH1 expression normalized to GAPDH and $\beta$-actin in the pCF depot of control, old, and Western diet-fed mice. (F) qPCR analysis of Adh1 expression in the pCF depot of control, old, and Western diet-fed mice. (C) qPCR analysis of ADH1 expression in the pCF depot of humans with a BMI below 30 or a BMI of 30 or higher. (H) qPCR analysis of $A D H 1$ expression in the pCF depot of humans aged 30 years or younger and 50 years or older. $n=3$ per group for biological animal replicates in the Western blot; $n=4-6$ per group for biological animal replicates for qPCR analysis; $n=5$ for samples from young and old humans; $n=6-8$ for samples from lean and obese humans. Data are presented as the mean \pm SEM. ${ }^{*} P=0.05$, by Student's $t$ test for comparisons between 2 groups for human data, or by 1-way ANOVA with Tukey's HSD multiple-comparison test for comparison of the mean of Western diet-fed mice and old mice with the mean of the control group.

of iVF and pCF. This analysis revealed a distinct molecular signature in $\mathrm{pCF}$ that was characterized by 2900 differentially regulated transcripts (Figure 2A and Supplemental Data File 1; supplemental material available online with this article; https://doi.org/10.1172/ JCI141799DS1). Pathway analysis further revealed significant regulation for molecules implicated in vitamin A (retinol) metabolism and retinoic acid (RA) signaling (Figure 2B). Among the enzymes capable of initiating the retinol metabolism cascade, $\mathrm{ADH} 1$ was the only one whose expression was identified by our transcriptomics analysis (Supplemental Data File 1). ADH1 is one of the enzymes responsible for facilitating the oxidation of retinol into retinaldehyde, which is then subsequently oxidized by aldehyde dehydrogenase 1 (ALDH1) to produce RA (Figure 2C). We specifically found that the $\mathrm{PCF}$ remodeling observed with aging and a Western diet was characterized by decreased levels of ADH1 (Figure 2, D-F). Also, expression analysis for other genes implicated in retinol metabolism showed additional changes, although the level of regulation was not as strong as that seen for Adh1 (Supplemental Figure 1). Importantly, we also found that in humans, $A D H 1$ expression was reduced in the pCF of individuals with obesity (Figure 2G), and we observed a similar trend in humans with aging (Figure 2H).

These results show that pCF increased in size and whitened in aged and Western diet-fed obese mice and that PCF accumulation correlated with cardiometabolic impairments. In addition, these data demonstrate that $\mathrm{PCF}$ was distinct from $\mathrm{iVF}$ and that human and mouse $A D H 1 / A d h 1$ expression was reduced in conditions characterized by $\mathrm{pCF}$ expansion such as aging and obesity.

$A D H 1$ regulates $p C F$ accumulation and remodeling. To determine whether ADH1 is a primary regulator of $\mathrm{pCF}$, we analyzed 4-month-old Adh1-KO mice and found specific expansion of $\mathrm{pCF}$ (Figure 3A). We further quantified this increase in pCF size by gravimetric assessment of $\mathrm{pCF}$ weight (Figure 3B). $\mathrm{pCF}$ expansion was the only obvious phenotype of Adh1-KO mice and occurred without a change in heart weight or body weight (BW) (Figure 3, $\mathrm{C}$ and $\mathrm{D})$. We observed no alterations in subcutaneous, intra-abdominal, or brown fat depots in Adh1-KO mice (Supplemental Fig- 
A
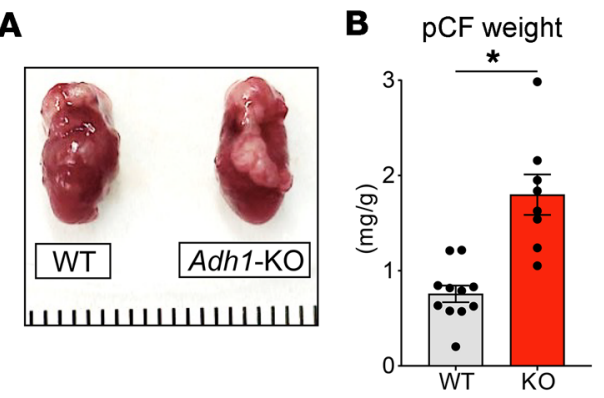

C Heart weight D

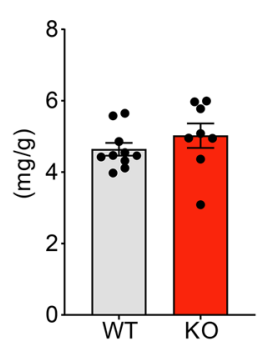

BW

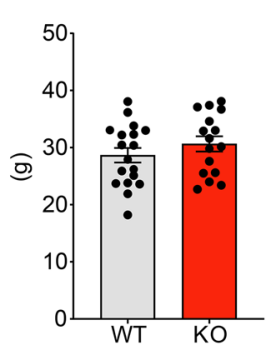

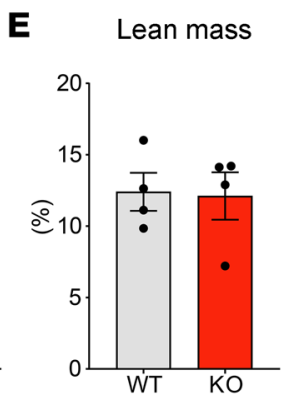

F Fat mass

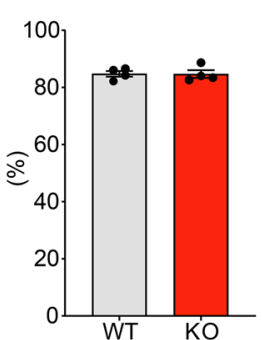

G

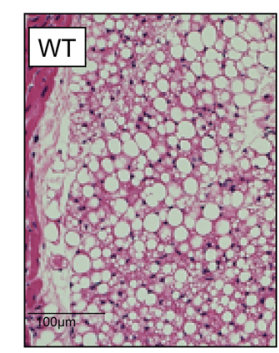

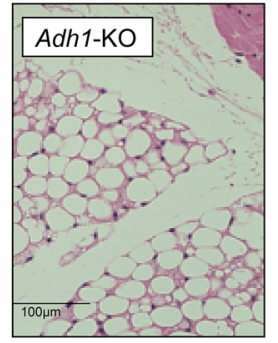

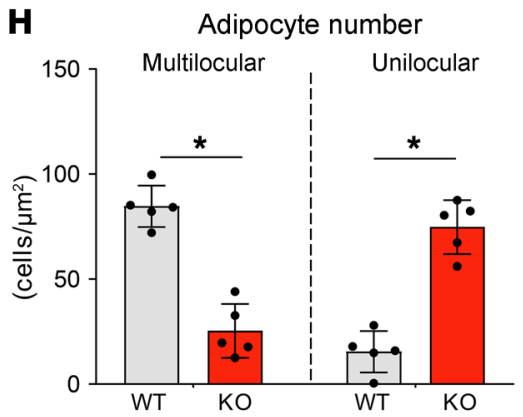

I Adipocyte size

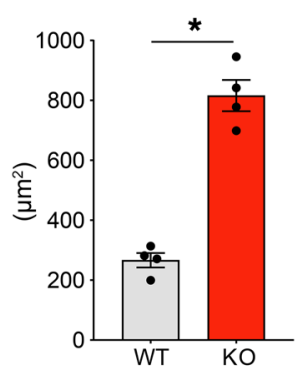

J

K

Activity

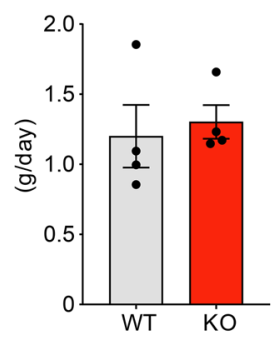

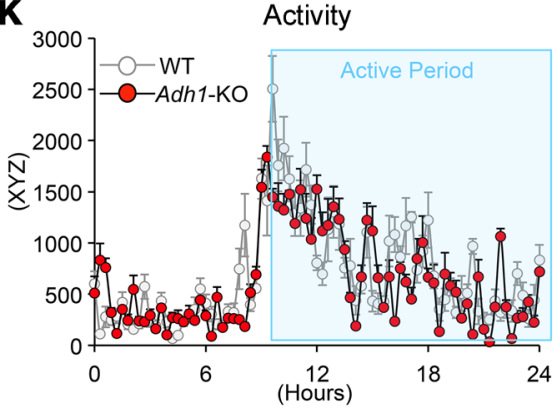

Fuel use

o

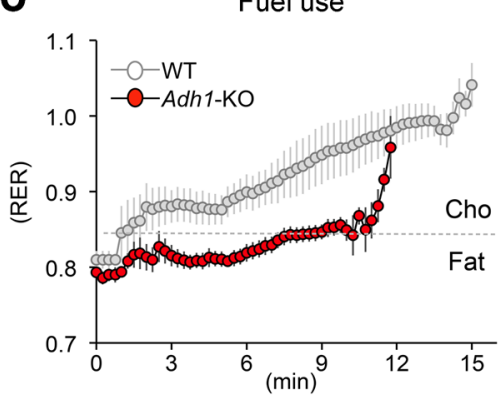

L Fasting glucose

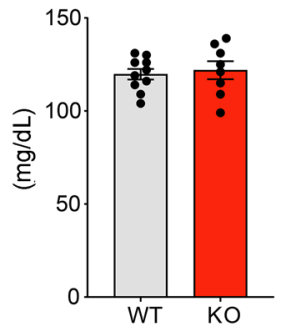

Calorimetry

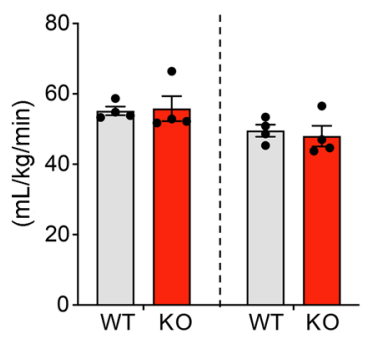

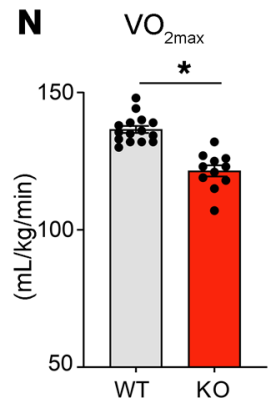

$\mathbf{P}$
Carbohydrate oxidation

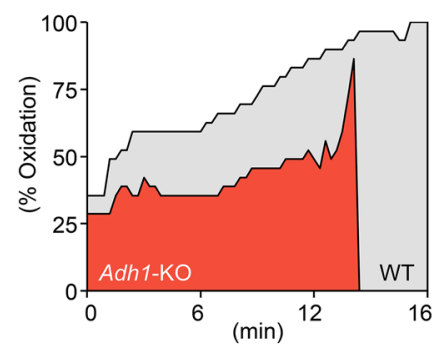

Q Time until

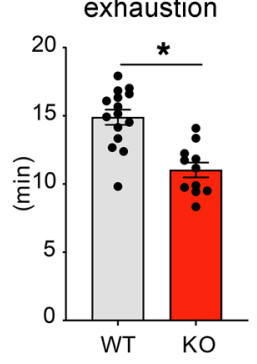

Figure 3. Adh1 deficiency drives pCF accumulation and impairments in cardiometabolic fitness. (A) Representative image of hearts with pCF from 4-monthold WT and Adh1-KO mice. (B and C) pCF and heart weights normalized to BW and (D) BW of the mice. (E and F) Measurements of lean mass and fat mass in WT and Adh1-KO mice using the total body EchoMRI analyzer. (C) Representative H\&E-stained images of the pCF depot from WT and Adh1-KO mice. Scale bars: $100 \mu \mathrm{m}$. (H) Distribution of multilocular and unilocular adipocytes and (I) unilocular adipocyte size in WT and Adh1-KO animals. (J) Food intake over a 24-hour period and (K) activity (XYZ; blue box indicates the awake period) for WT and Adh1-KO animals. (L) Overnight fasting glucose and (M) average 24-hour oxygen consumption and $\mathrm{CO}_{2}$ production in WT and Adh1-KO animals. (N) $\mathrm{VO}_{2 \max }$ (maximal oxygen consumption), (0)RER (a measure of fuel substrate use), (P) carbohydrate (Cho) oxidation, and (Q) time until exhaustion during graded maximal exercise testing. $n=4-15$ per group for biological animal replicates; $n$ $=160-800$ cells for adipocyte quantification; and 50-100 cells for adipocyte size quantification for each biological replicate. Data are presented as the mean \pm SEM for the bar graphs. The dots in $\mathbf{K}$ and $\mathbf{O}$ indicate the mean points over the course of a test. ${ }^{*} P=0.05$, by Student's $t$ test.

ure 2). In agreement with these data, we found that the overall percentage of lean and fat mass was also unchanged in the absence of ADH1 (Figure 2, E and F). To determine whether the loss of ADH1 alters pCF phenotypic characteristics, as observed with models of cardiometabolic impairments characterized by pCF accumula- tion such as aging and a Western diet, we performed a histologic analysis of pCF from WT and Adh1-KO mice. The pCF of Adh1KO mice had visible "whitening" of the depot, characterized by an increased percentage of unilocular versus multilocular adipocytes, as well as unilocular adipocyte hypertrophy (Figure 3, G-I). 
A Metabolic pathway analysis

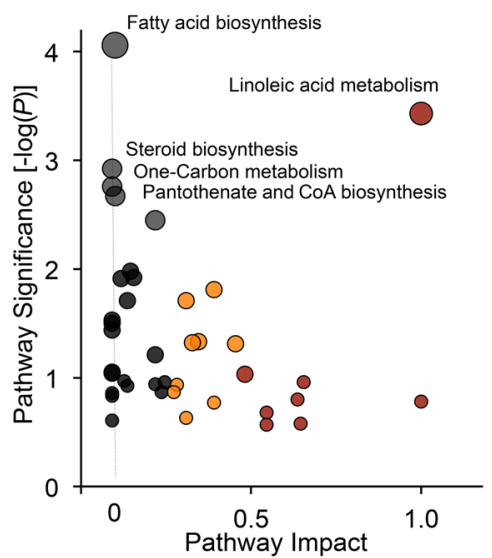

B

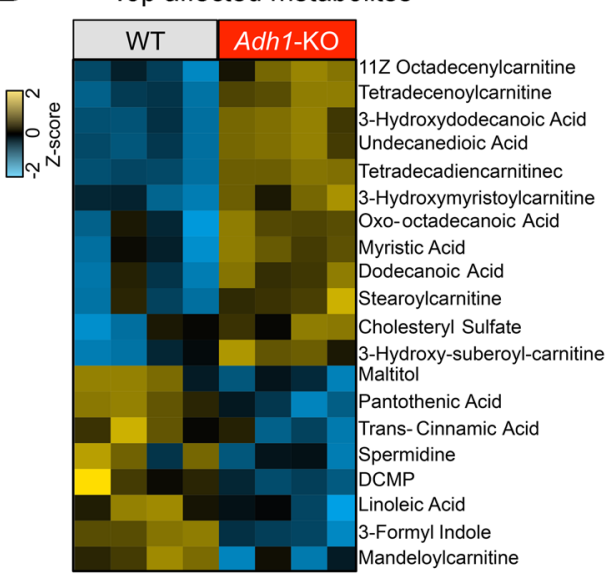

C

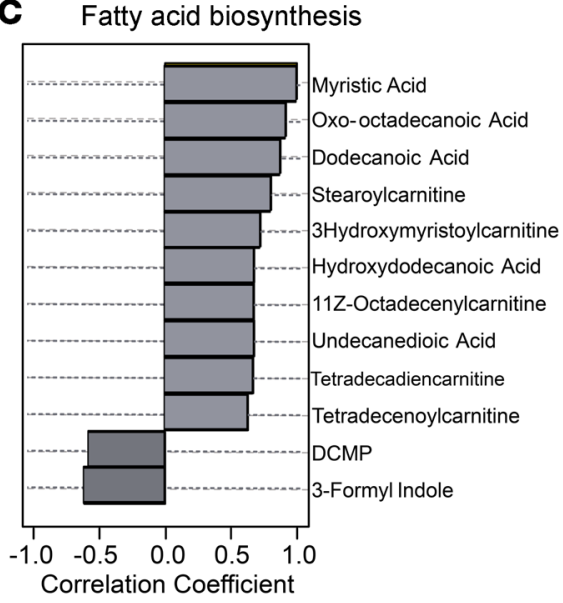

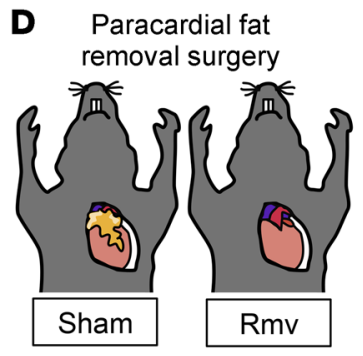

E

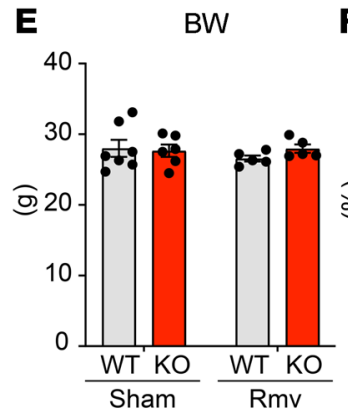

F

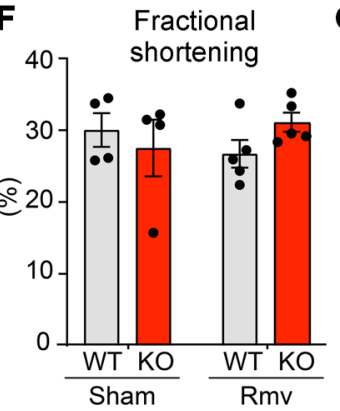

G
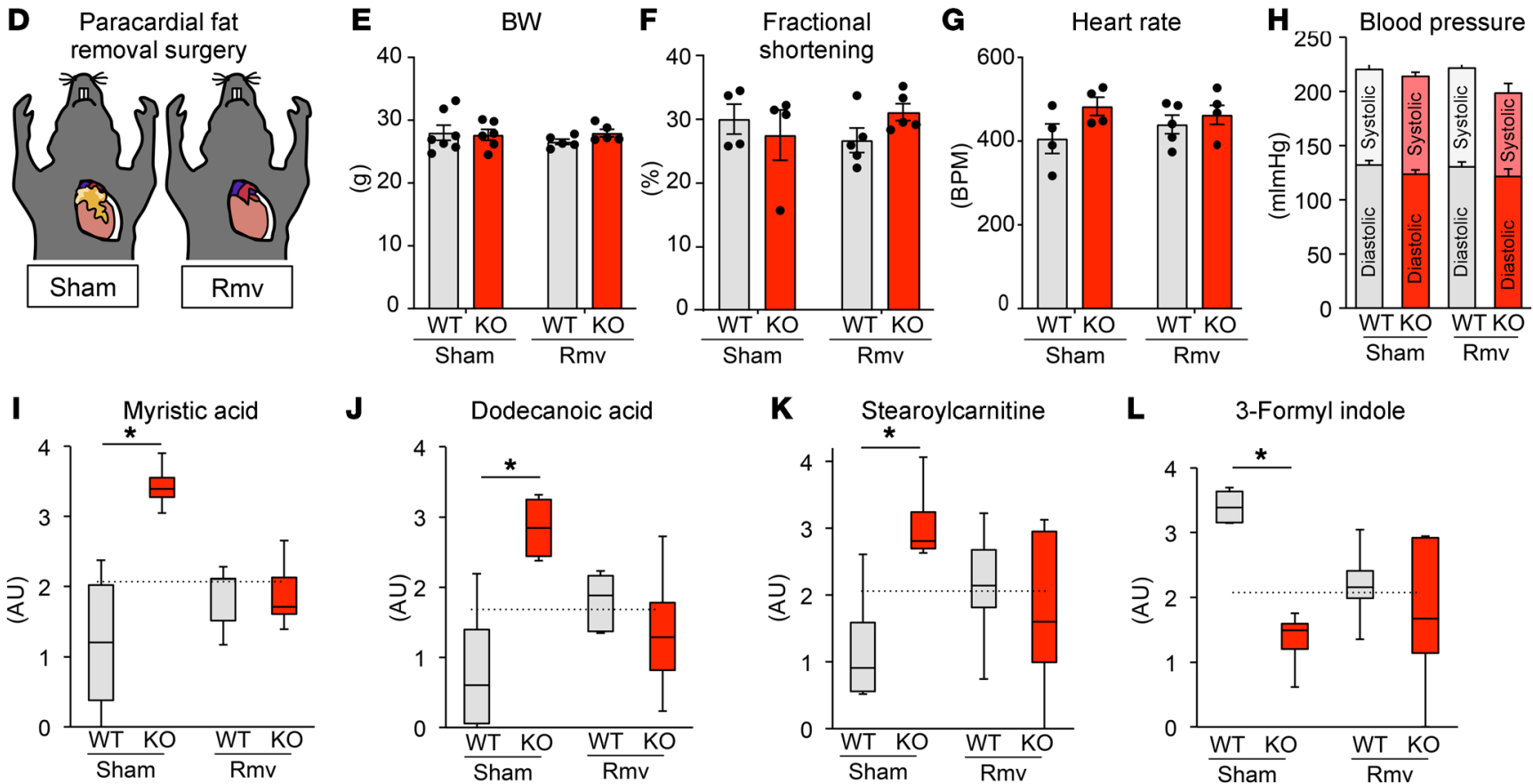

J

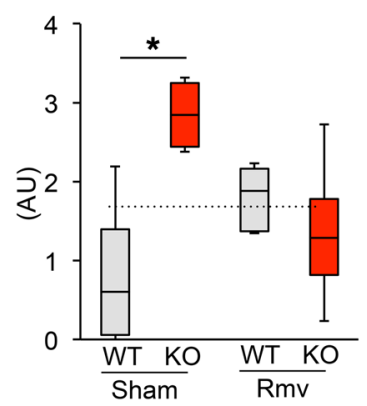

K Stearoylcarnitine
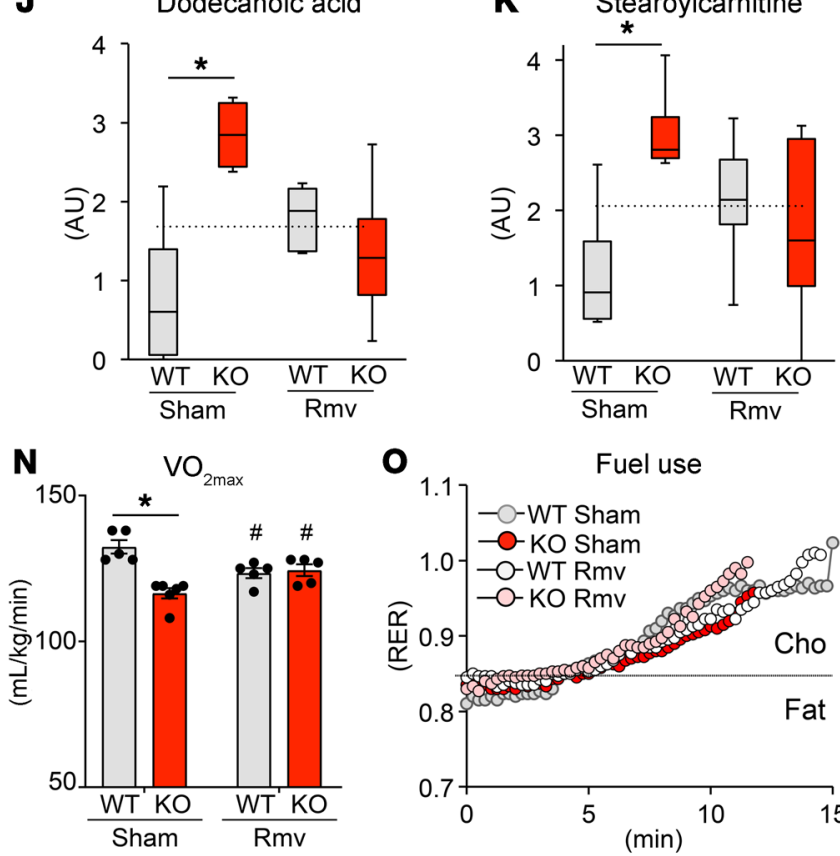

Fuel use

0

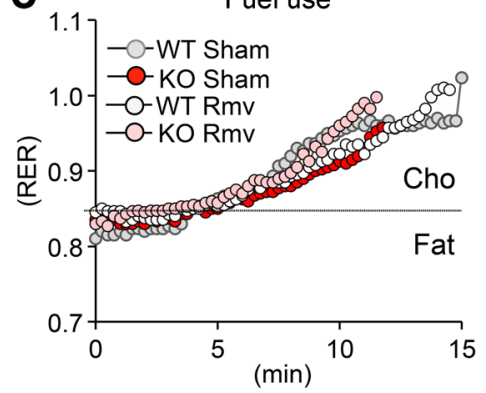

$\mathbf{L}$

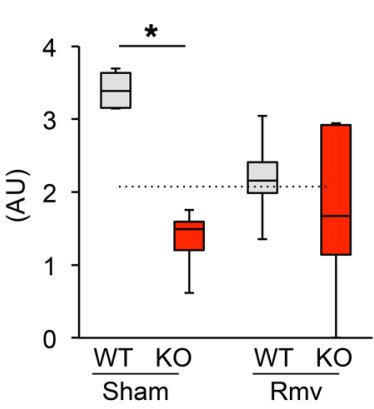

P Carbohydrate oxidation

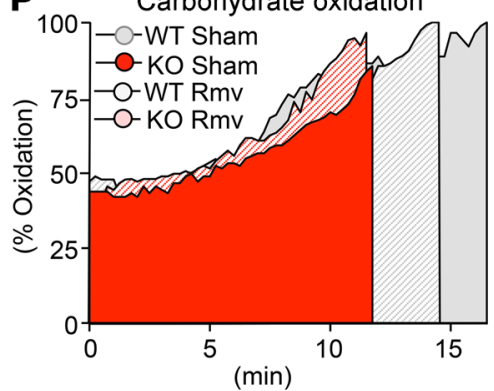

Figure 4. pCF regulates circulating metabolites and cardiometabolic fitness. (A) Metabolomics pathway analysis of differentially regulated metabolites in plasma from 4-month-old WT and Adh1-KO. (B) Heatmap of top 20 significantly different metabolites in the plasma of WT and Adh1-KO animals. (C) List of metabolites that correlate with fatty acid biosynthesis. (D) Representative schematic of pCF removal (Rmv) and sham surgeries. (E) BW, (F) echocardiographic percentage of fractional shortening, (C) heart rate, and (H) blood pressure measurements after sham or pCF removal surgery in 4-month-old WT and Adh1-KO mice. (I-L) Relative levels of top metabolites implicated in fatty acid biosynthesis in WT and Adh1-KO plasma following sham or pCF removal surgery. (M) Time until exhaustion and (N) $\mathrm{VO}_{2 \max }$ during graded maximal exercise testing. (0) RER during graded maximal exercise testing for the WT and Adh1-KO sham-operated and pCF removal groups. (P) Carbohydrate oxidation during graded exercise testing for WT and Adh1-KO sham-operated and pCF removal groups. $n=4-7$ per group for biological animal replicates. Data are presented as the mean \pm SEM for bar graphs, as dot plots indicating the mean over the course of a test, as $2 \mathrm{D}$ area charts for oxidation graphs, or as box-and-whisker plots. Data were analyzed by Student's $t$ test for comparisons between 2 groups or by 2-way ANOVA with Tukey's HSD multiple-comparison test for consideration of both treatments and genotypes. Asterisks indicate significance between genotypes of the same group (sham-operated, pCF removal), and the pound signs indicate significance within the same genotype but with different treatments, at $P=0.05$. 
A

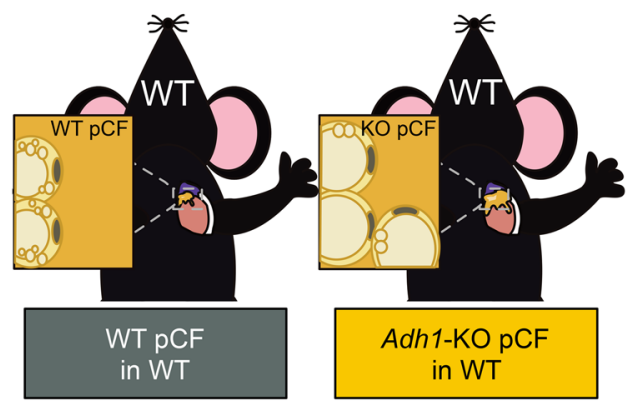

E

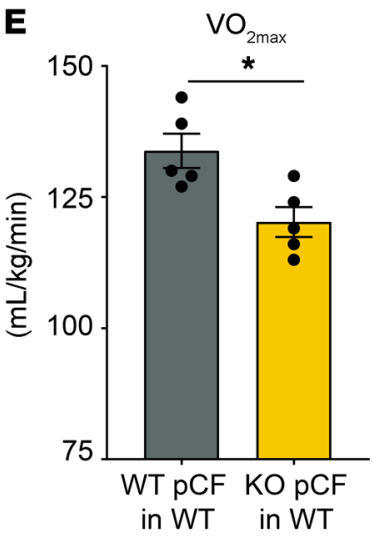

H

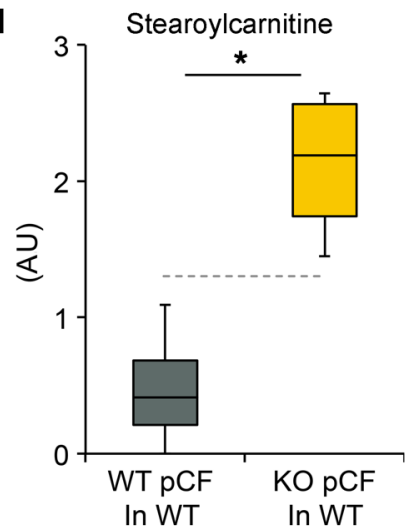

B

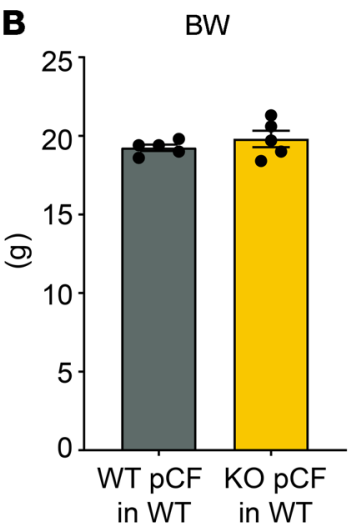

F

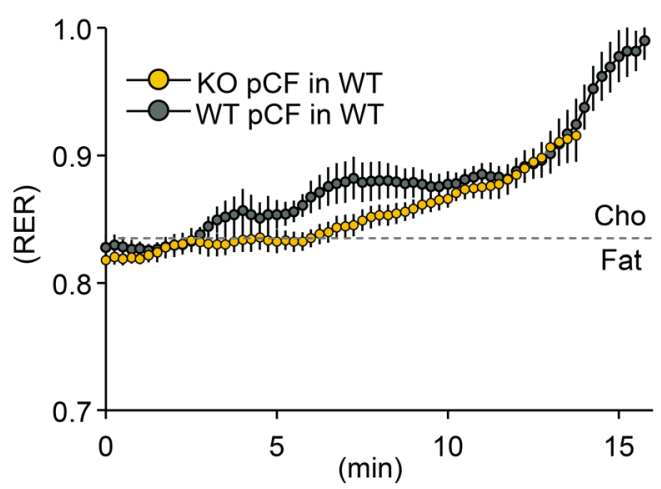

Fuel use
C

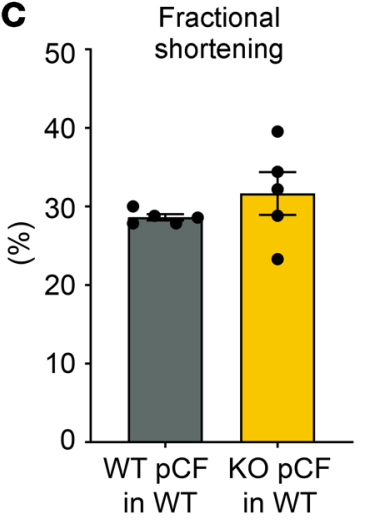

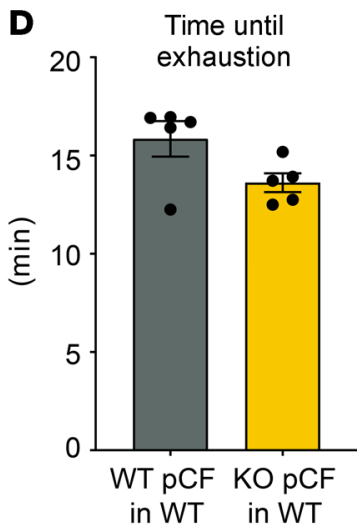

G

Carbohydrate oxidation
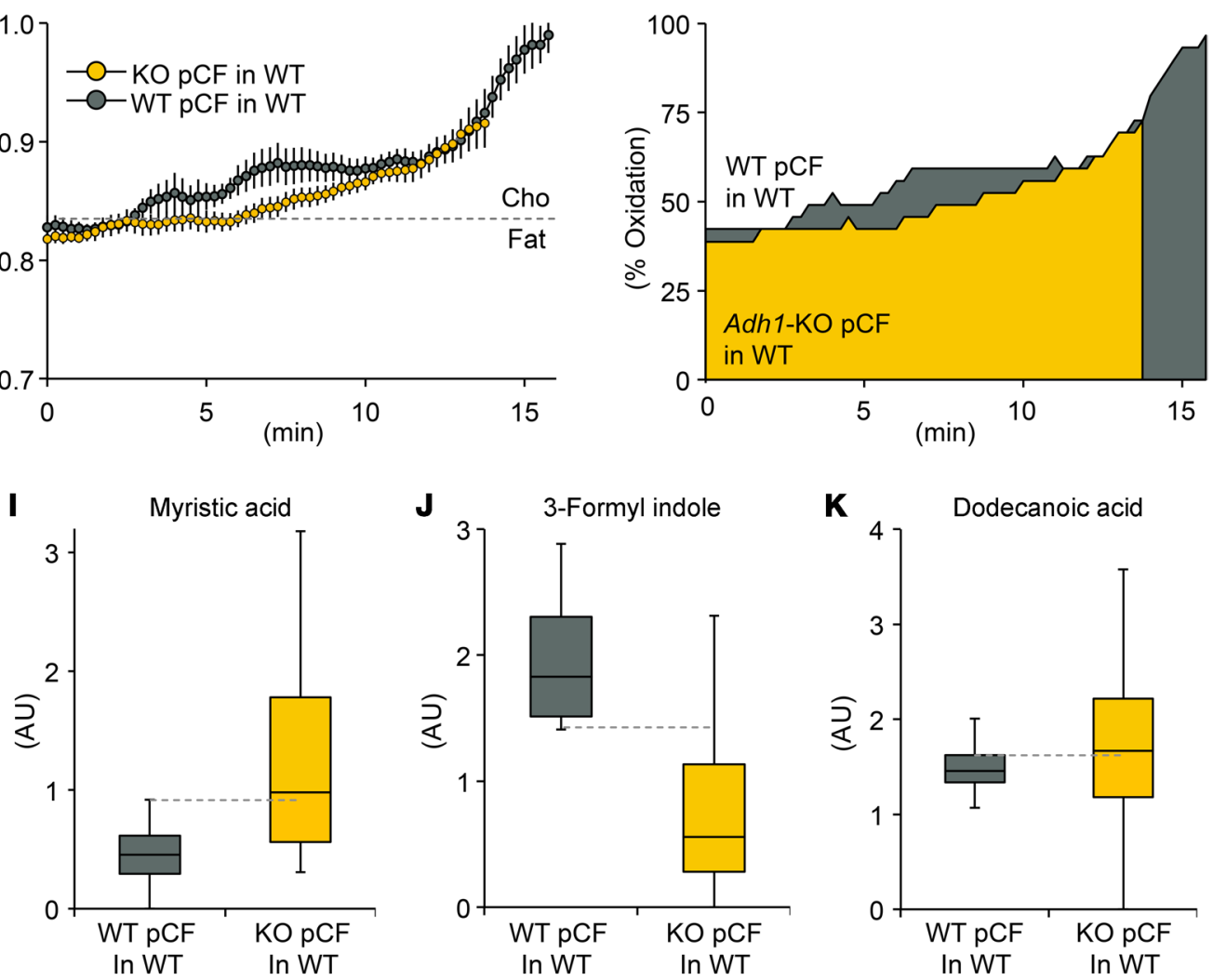

Figure 5. pCF transplantation recapitulates the Adh1-KO phenotype. (A) Representative schematic of pCF transplantation surgeries. (B) BW and (C) echocardiographic percentage of fractional shortening for 4-month-old WT mice transplanted with WT pCF or Adh1-KO pCF. (D) Time until exhaustion and (E) $\mathrm{VO}_{2 \max }$ during graded maximal exercise testing. (F) RER during graded maximal exercise testing of WT mice that received WT pCF or Adh1-KO pCF transplants. (C) Carbohydrate oxidation during graded maximal exercise testing for WT pCF and Adh1-KO pCF transplant groups. (D-K) Relative levels of the indicated metabolites in WT mice that received WT pCF or Adh1-KO pCF transplants. $n=5$ per group for each biological replicate. Data are presented as the mean \pm SEM for bar graphs, as dot plots indicating the mean over the course of a test, as $2 \mathrm{D}$ area charts for oxidation graphs, or as box-and-whisker plots. ${ }^{*} P=0.05$, by Student's $t$ test.

To assess whether the absence of ADH1 altered mouse behavior, we then tested food intake and activity and observed no changes in these parameters in Adh1-KO mice compared with controls (Figure 3, J and K). Additionally, fasting glucose levels and baseline calorimetric analysis revealed that no alterations occurred with genetic ablation of Adh1 (Figure 3, L and M). Interestingly, when subjected to graded maximal exercise testing, Adh1-KO mice showed impaired performance. In this test, relative oxygen con- sumption and power output increase with each stage and thus promote a shift from fat to predominantly carbohydrate utilization to support the increasing ATP demand of increased exercise intensity. This analysis revealed that the Adh1-KO animals had impaired performance when the test intensity increased and carbohydrate metabolism was predominant, which resulted in decreased maximal oxygen consumption $\left(\mathrm{VO}_{2 \max }\right)$ and impaired carbohydrate utilization (Figure 3, N-P). The consequent reduced running time 
A

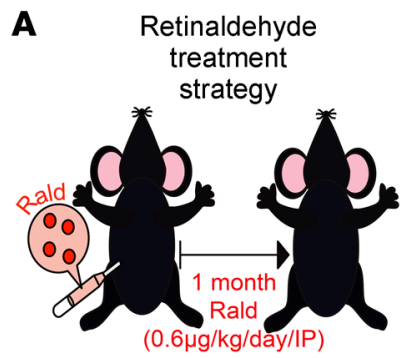

B

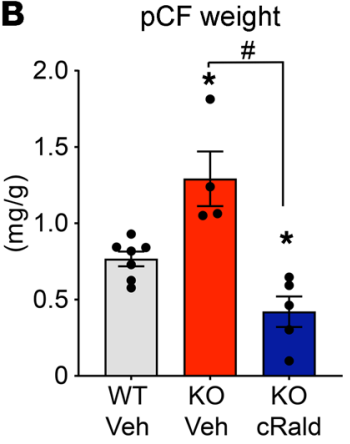

$\mathbf{F}$

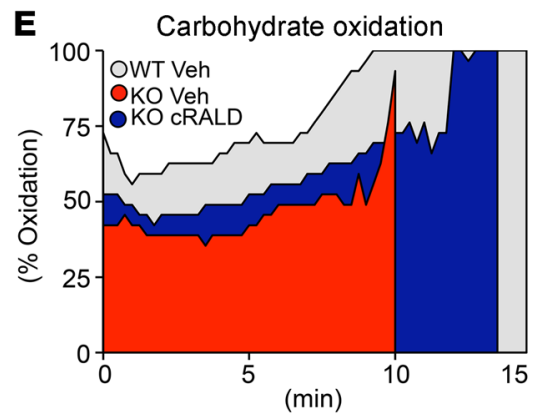

C

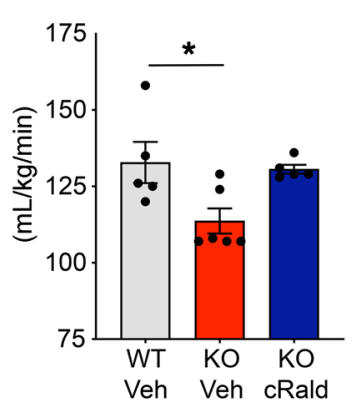

H\&E

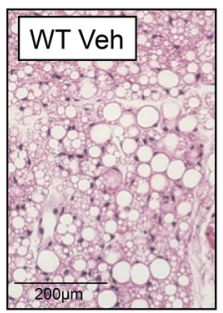

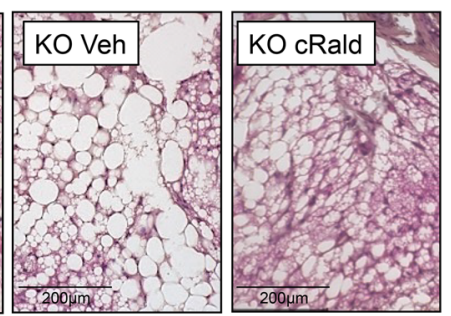

D

Fuel use

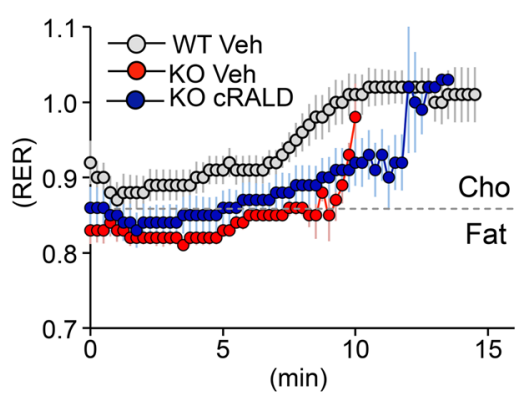

Figure 6. Retinaldehyde treatment rescues pCF remodeling and metabolic disorder in Adh1-KO mice. (A) Schematic of daily i.p. retinaldehyde (Rald) injections. (B) pCF weight normalized to BW for the indicated genotypes and treatments. Veh, vehicle. (C) $\mathrm{VO}_{2 \max }$ (D) RER, and (E) carbohydrate oxidation in WT vehicle-treated, Adh1-KO vehicle-treated, and Adh1-KO mice treated with retinaldehyde for 1 month (chronic retinaldehyde [cRald]). (F) Representative $\mathrm{H} \& \mathrm{E}$-stained images of pCF from mice of the indicated genotypes and treatment groups. (G) qPCR quantification of relative mtDNA abundance normalized to nuclear DNA (nDNA) content. $n=4-7$ per group for each biological replicate. Data are presented as the mean \pm SEM for bar graphs, as dot plots indicating mean points over the course of a test, and as $2 \mathrm{D}$ area charts for oxidation graphs. Data were analyzed by 1-way ANOVA with Tukey's HSD multiple-comparison test. Asterisks indicate significance compared with the WT vehicle-treated group and the pound symbol indicates significance between Adh1-KO vehicle and Adh1-KO chronic retinaldehyde treatment groups at a significance level of $P=0.05$.

indicated exercise intolerance (Figure 3Q). The observed running impairment occurred in the absence of an overt skeletal muscle, liver, or heart phenotype (Supplemental Figures 3 and 4 ). The lack of alterations in these major organs is likely explained by unaltered circulating global levels of RA in Adh1-KO mice (Supplemental Figure 5A). However, as defects in converting retinol to retinaldehyde can results in alternative transformation of retinol into retinyl esters, we confirmed that, indeed, the absence of ADH1 led to an accumulation of retinyl esters, while we observed only a trend toward retinol accumulation (Supplemental Figure 5, B and C). $\mathrm{ADH} 1$-mediated regulation of $\mathrm{pCF}$ appeared to be independent from expression of uncoupling protein 1 (UCP1) as neither Adh1KO pCF nor acute knockdown of Adh1 in adipogenic cells affected Ucp1 levels (Supplemental Figure 5, D-F). However, ADH1 loss ofunction was sufficient to affect the expression of genes implicated in adipocyte biology such as PR domain-containing 16 (Prdm16), whose ablation was sufficient to drive a "beige-to-white" switch in adipocytes (Supplemental Figure 5, E and F).

Hence, mice lacking ADH1 accumulated pCF and displayed metabolic impairments during exercise, in which they failed to switch to a predominant carbohydrate metabolism to support increasing ATP demands, leading to exercise intolerance. Also, Adh1 ablation drove pCF-specific whitening, suggesting that $\mathrm{ADH1}$ is an overall critical regulator of pCF remodeling.

PCF removal normalizes the Adh1 deficiency phenotype. The cardiometabolic defects we observed in Adh1-KO mice upon exercise stress hinted at systemic metabolic alterations in these mice. To test whether the absence of ADH1 indeed affects circulating metabolites, we performed an unbiased metabolomics analysis of plasma samples from 4-month-old mice. This experiment revealed alterations in the plasma concentrations of several metabolites, with fatty acid biosynthesis being the most significantly affected pathway (Figure 4A). More specifically, among the top 20 most significantly changed metabolites between WT and Adh1-KO plasma were 12 molecules implicated in fatty acid biosynthesis (Figure 4, B and C).

To definitively prove the dependency of the metabolic phenotypes observed in Adh1-KO mice upon pCF remodeling, we established an open-heart surgical procedure that allowed us to remove the pCF depot (Figure 4D). Four weeks after the surgery, we assessed the effects of $\mathrm{PCF}$ removal or sham surgeries on WT and Adh1-deficient mice. Removal of pCF did not alter BW (Figure $4 \mathrm{E}$ ), and echocardiographic assessment of cardiac function by fractional shortening revealed no alterations following $\mathrm{pCF}$ removal or genetic ablation of Adh1 (Figure 4F). Similarly, we observed no alterations in heart rate or blood pressure following sham or pCF removal surgery (Figure $4, G$ and $H$ ). To assess the role of pCF in regulating systemic metabolism, we performed metabolomics screenings of plasma samples from sham controls and mice with PCF removal in both WT and Adh1-KO genotypes. This unbiased analysis revealed that the differences in fatty acid metabolites observed in Adh1-deficient mice were largely 
A
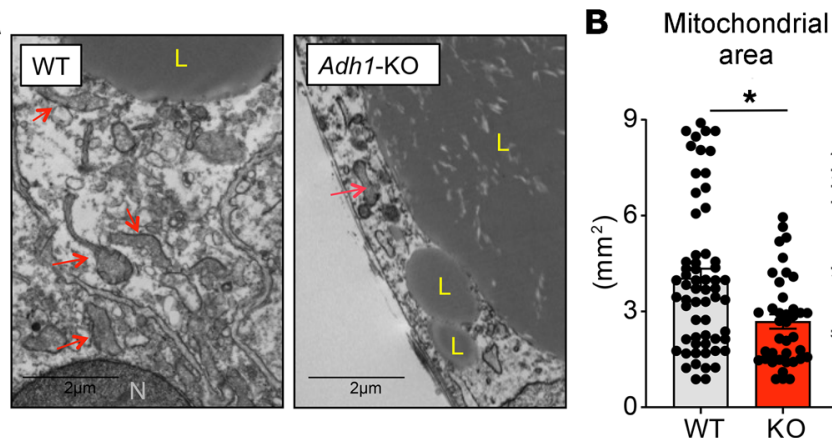

D

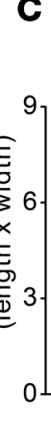

Aspect

ratio

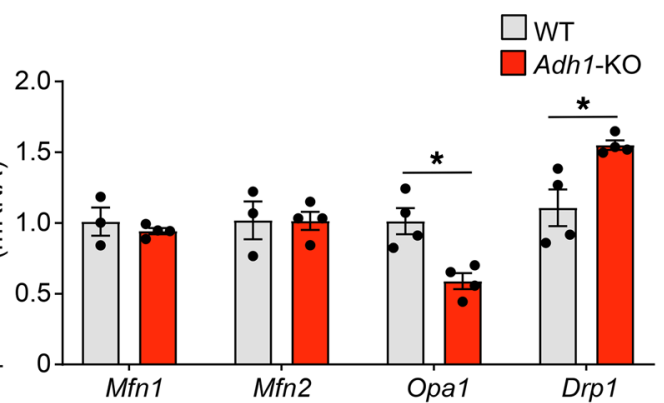

E

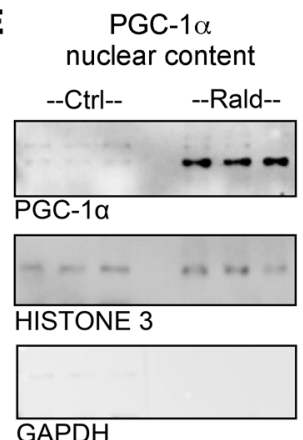

F PGC- $1 \alpha$ Iocalization PGC-1 $\alpha$ |DAPI| Merge
G

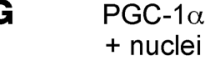

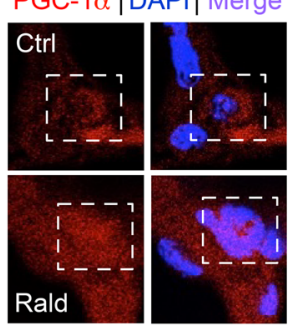

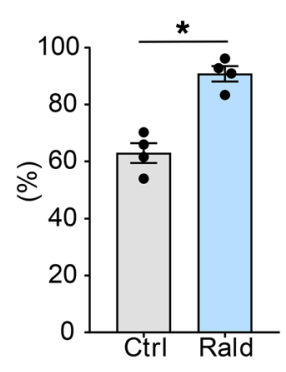

H

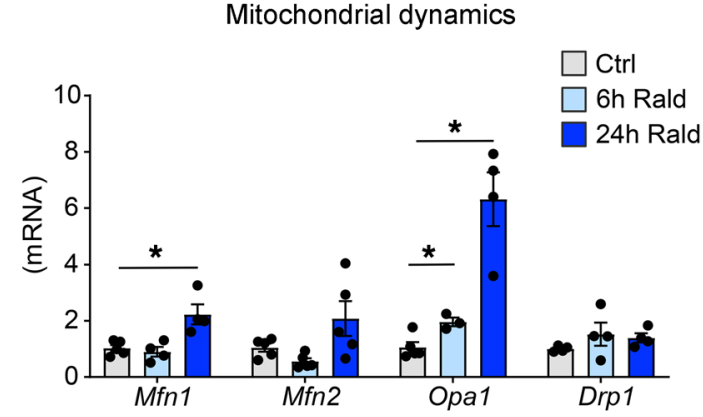

I

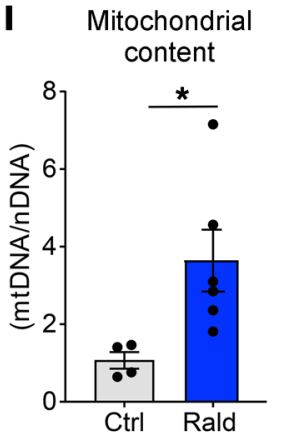

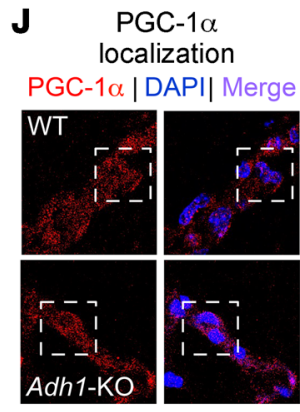

K PGC-1 $\alpha$

+ nuclei
$\mathbf{L}$

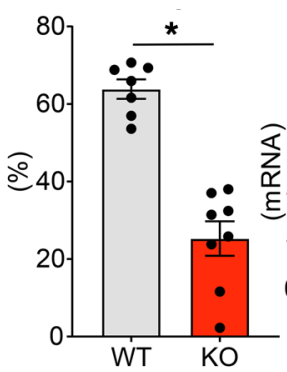

$\mathbf{M}$

Mouse
Opa1

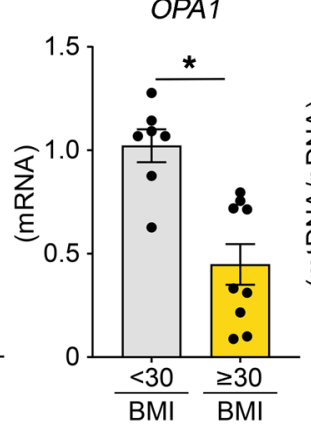

N Human mitochondrial content

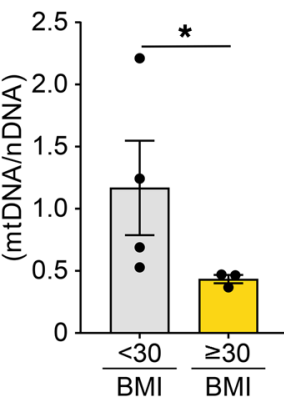

Figure 7. The ADH1/retinaldehyde pathway regulates pCF remodeling by altering mitochondria through PGC-10. (A) Representative TEM images of pCF from WT and Adh1-KO mice. L, lipid droplet; N, nucleus. Arrows indicate mitochondria. Scale bars: $2 \mu \mathrm{m}$. (B) Measurements of mitochondrial area and (C) aspect ratio from TEM images of pCF from WT and Adh1-KO mice. (D) qPCR for mitofusin 1 (Mfn1), mitofusin 2 (Mfn2), Opa1, and Drp1 mRNA expression in pCF. (E) PCC-1 $\alpha$ protein expression in nuclear pCF extracts isolated from WT animals injected systemically with vehicle or retinaldehyde for 6 hours compared with expression in the nuclear protein loading control. (F) Immunofluorescence analysis of PGC- $1 \alpha$ (red) in pCF from WT controls or mice treated for 6 hours with retinaldehyde and (C) quantification of PGC-1 $\alpha$-positive nuclei from the immunofluorescence images in F. Original magnification, $\times 400$. (H) qPCR analysis of expression of the indicated genes in pCF following vehicle or retinaldehyde treatment for 6 hours or 24 hours. (I) qPCR analysis of mtDNA abundance in pCF following 24 hours of treatment with retinaldehyde or vehicle control in WT mice. (J) Immunofluorescence analysis of PGC-1 $\alpha$ (red) in WT or Adh1-KO pCF and (K) quantification of PGC-1 $\alpha$-positive nuclei in immunofluorescence images of WT or Adh1-KO pCF. (L) qPCR analysis of Opa1 expression in the pCF depot from WT vehicle-treated, Adh1-KO vehicle-treated, and Adh1-KO chronic retinaldehyde-treated mice (1-month-long treatments). (M) qPCR analysis of OPA1 expression in the pCF depot of humans with a BMI below 30 or a BMI of 30 or higher. (N) qPCR quantification of relative mtDNA abundance normalized to nDNA content in the pCF depot of humans with a BMI below or above 30. $n=7-9$ per group for human gene expression analysis; $n=3-4$ for mitochondrial content measurements; $n=3-5$ per group for mouse gene expression analysis; $n=4-6$ per group for mouse mitochondrial content measurements; $n=4-8$ per group for animal biological replicates for immunostaining quantification; and $n \geq 65$ nuclei analyzed per biological replicate for TEM quantifications. Data are presented as the mean \pm SEM for bar graphs. Data were analyzed by Student's $t$ test when comparing 2 groups or by 1-way ANOVA with Tukey's HSD multiple-comparison test for comparisons of 3 groups. Asterisks indicate significance compared with the control group, and the pound symbol indicates significance between Adh1-KO vehicle-treated and Adh1-KO chronic retinaldehyde-treated groups at $P=0.05$.

normalized when pCF was removed (Figure 4, I-L, and Supplemental Figure 6, A-G). To better understand the nature of the metabolic changes driven by the absence of ADH1 in a pCF-dependent manner, we then analyzed fatty acid plasma content on the basis of their carbon chain length. Although specific changes in medium and long chain fatty acids were observed in the absence of ADH1, we detected normalization of these changes with pCF removal in Adh1-KO plasma compared with WT plasma (Supplemental Figure 6I). However, total free fatty acid content was not changed by either genetic deletion of $A d h 1$ or pCF removal (Supplemental Figure 6H). Overall, metabolomics analysis uncovered systemic ADH1-mediated, pCF-dependent effects that could underlie the cardiometabolics impairments we observed in the Adh1-KO mice. 
To determine the importance of pCF for the cardiometabolic alterations observed in Adh1-deficient mice, we then subjected mice lacking pCF to graded maximal exercise testing (Figure 4, $\mathrm{M}-\mathrm{P})$. Sham Adh1-KO animals showed the expected reduction in $\mathrm{VO}_{2 \max }$, and removal of pCF normalized their $\mathrm{VO}_{2 \max }$ values to that of their WT counterparts (Figure $4 \mathrm{~N}$ ). Interestingly, pCF removal appeared to negatively impact $\mathrm{VO}_{2 \max }$ in the WT group, suggesting a physiological role for healthy pCF during cardiometabolic stress (Figure $4 \mathrm{~N}$ ). Fuel use analysis during exercise testing also revealed improvement in the ability of Adh1-KO mice to use carbohydrates as a fuel source when $\mathrm{pCF}$ was removed (Figure 4, O and $\mathrm{P}$ ).

Together, these data revealed the importance of $\mathrm{pCF}$ for the phenotypes observed in Adh1-deficient mice and a role for this depot in the regulation of circulating metabolites.

Transplantation of remodeled $p C F$ recapitulates the Adh1 deficiency phenotype. The results obtained by our pCF removal study suggested the important contribution of this depot for the phenotypes observed in our Adh1-null mice. However, removal of pCF from healthy control mice had an impact on cardiometabolic fitness that could have confounded some of the rescue results observed when assessing genotype effects following pCF removal. For this reason, we decided to conduct an independent experiment in which the contribution of pCF to cardiometabolic fitness and circulating metabolites was assessed through transplantation surgeries. To definitively assess how pCF from Adh1-deficient mice affects cardiometabolics, pCF from Adh1-KO mice was transplanted into WT mice to replace their endogenous pCF (Adh1-KO pCF in the WT group); as a control, we removed pCF from WT mice and transplanted it into a different cohort of WT mice in place of their original pCF (WT pCF in the WT group) (Figure 5A). The transplanted mice were then analyzed 4 weeks after recovery from the surgery. As expected, based on our previous results for pCF removal, we found that the transplantation procedure had no effect on BW or cardiac function (Figure 5, B and C). However, graded maximal exercise testing showed that, although the time until exhaustion was not significantly affected, $\mathrm{VO}_{2 \max }$ was impaired in the mice that received Adh1-KO pCF (Figure 5, D and E). Importantly, these effects were not dependent on the surgical procedure itself, as transplantation of WT pCF into WT mice did not affect these parameters (Supplemental Figure 7). Fuel utilization analysis also revealed that transplantation of Adh1-KO pCF into WT mice reduced their reduced ability to utilize carbohydrate as a fuel during exercise stress testing compared with WT mice that received WT pCF (Figure 5, F and G). These results demonstrated that $\mathrm{pCF}$ from $A d h 1-\mathrm{KO}$ mice was sufficient to reproduce the cardiometabolic defects observed in mice with global $A d h 1 \mathrm{KO}$ and highlight how pCF remodeling can act as a central regulator of cardiometabolic fitness.

We next performed plasma metabolomics to assess which circulating metabolites were directly affected by Adh1-KO pCF transplantation (Figure 5, H-K). From this unbiased analysis, we found that stearoylcarnitine, a key molecule that was altered in the plasma of global Adh1-KO mice, was also altered in WT mice that received $A d h 1-\mathrm{KO}$ pCF transplants (Figure $5 \mathrm{H}$ ). Other metabolites that were altered in the plasma of whole-animal Adh1 KOs showed a similar trend toward an increase (myristic acid) or decrease (3-formyl indole) with Adh1-KO pCF transplantation in WT mice
(Figure 5, I and J). Dodecanoic acid levels were, on the other hand, unchanged (Figure 5K). Overall, the results demonstrated that transplantation of Adh1-KO pCF into WT animals was sufficient to reproduce the cardiometabolic defects observed in mice with global Adh1 KO. These results point to pCF remodeling as central for the regulation of cardiometabolic fitness.

Retinaldehyde treatment rescues $p C F$ remodeling and metabolic disorder in Adh1-KO mice. Our pCF removal studies demonstrated the importance of $\mathrm{ADH} 1$ in the regulation of this specific fat depot. The fact that the loss of $\mathrm{ADH} 1$ is maladaptive for $\mathrm{pCF}$ function also points to its downstream product retinaldehyde as a regulator of $\mathrm{pCF}$ physiology. $\mathrm{ADH} 1$ primarily catalyzes oxidation of retinol to retinaldehyde; however, we could not exclude the possibility that its loss might generate secondary compensatory changes that could confound the retinaldehyde-dependent effects on pCF in vivo. To investigate whether the defect observed in the pCF of Adh1-deficient mice was truly due to a reduced capacity to generate retinaldehyde, we performed daily retinaldehyde injections for 1 month until the animals reached 4 months of age (chronic retinaldehyde treatment) (Figure 6A). We found that systemic retinaldehyde treatment completely rescued the accumulation of pCF observed in Adh1-KO mice (Figure 6B). Additionally, the observed exercise-induced metabolic defect seen in Adh1-KO mice was reversed by chronic retinaldehyde treatment (Figure 6, C-E). Histological analysis revealed that the whitening of pCF observed in Adh1-KO mice was also reversed (Figure 6F). Importantly, the retinaldehyde regimen adopted in our experiments, which has proven to be beneficial in conditions of pathologic pCF remodeling such as that seen in Adh1-KO mice, did not lead to any overt alteration in healthy WT control mice (Supplemental Figure 8). Because mitochondrial content is key to distinguishing white and brown fat, we then measured mitochondrial content in these animals. Measurements of mitochondrial DNA (mtDNA) content showed that pCF from Adh1-KO mice had reduced mitochondrial content, which was rescued by retinaldehyde treatment (Figure 6G). This result demonstrated that ADH1's product retinaldehyde is an important regulator of $\mathrm{pCF}$ biology and prompted us to look more closely at the ADH1-retinaldehyde-related mechanism underlying mitochondrial regulation in the $\mathrm{pCF}$ depot.

The ADH1/retinaldehyde pathway regulates $p C F$ remodeling by altering mitochondria through PGC-1 $\alpha$. Mitochondrial dysfunction is associated with adipose tissue accumulation, as it has been shown that mitochondrial content declines as adipose tissue expands (9). In patients with obesity, declines in adipose mitochondrial content have been associated with the expansion of white, unilocular adipocytes $(29,30)$. To further determine whether defects in mitochondria underlay the pCF phenotype observed in Adh1-KO mice, we performed molecular and structural mitochondrial analysis. In-depth mitochondrial analysis in 4-monthold Adh1-KO mice using electron microscopy revealed reductions in mitochondrial area and aspect ratio, the latter being indicative of impaired mitochondrial fusion (Figure 7, A-C). The observed mitochondrial phenotype was supported by altered expression of mitochondrial dynamics molecules, with reduced expression of the fusion molecule optic atrophy 1 (Opa1) and increased expression of the fission molecule dynamin-related protein 1 (Drp1) 
(Figure 7D). Thus, $A d h 1$ ablation resulted in altered mitochondrial content and expression of key genes involved in mitochondrial dynamics within the pCF depot.

$\mathrm{ADH} 1$ is largely responsible for the generation of retinaldehyde, a molecule that can act as a direct active metabolite (31-35). To investigate the mechanism driving the mitochondrial defects observed in the pCF depot of Adh1-KO mice, we acutely treated WT mice systemically with retinaldehyde and analyzed its function in $\mathrm{pCF}$. Considering the central role of PPAR $\gamma$ coactivator $1 \alpha(\mathrm{PGC}-1 \alpha)$ as a master regulator of mitochondrial biogenesis and dynamics, we began by assessing whether retinaldehyde acts through this pathway in the pCF depot. Strikingly, we found that 6 hours of acute systemic retinaldehyde treatment resulted in nuclear translocation of PGC- $1 \alpha$ in the pCF depot (Figure 7, $\mathrm{E}-\mathrm{G})$. Interestingly, analysis of mitochondrial fission and fusion target genes revealed that Opa1 was the only transcript to be, and then stay, induced 6 and then 24 hours after retinaldehyde treatment (Figure $7 \mathrm{H})$. Consequently, in line with a role for PGC$1 \alpha$ in mitochondrial biogenesis, we found that retinaldehyde treatment was efficient at increasing pCF mitochondrial content within 24 hours of treatment (Figure 7I). Additionally, in agreement with our mechanistic findings, PGC-1 $\alpha$ nuclear translocation was reduced in Adh1-KO pCF (Figure 7, J and K), and, consequently, Opa1 expression was also reduced in $\mathrm{pCF}$ from Adh1-KO mice and strongly activated by chronic retinaldehyde treatment (Figure 7L). Together, these data suggest that retinaldehyde promotes PGC- $1 \alpha$ nuclear translocation to drive transcriptional activation of mitochondrial fusion genes, highlighting the importance of mitochondria and Opa1 expression for the phenotypic rescue of Adh1-KO pCF and the physiological function of pCF adipocytes in general. Importantly, the translational potential of these findings were elucidated, as both OPA1 expression and mitochondrial content were significantly downregulated in human pCF samples from individuals with obesity (Figure 7, M and $\mathrm{N}$ ). These data overall suggest the evolutionary importance of $\mathrm{ADH} 1$ and retinaldehyde production for the preservation of pCF homeostasis through the maintenance of mitochondrial mass, morphology, and abundance.

\section{Discussion}

Understanding the physiological role of the pCF depot, its functions, and the determinants that drive its accumulation is crucial for ultimately understanding its relationship with obesity and cardiovascular disease (refs. 25, 36 and reviewed in refs. 37-39). Adipose depots have multiple functions, in that they not only store lipids, but also signal and elicit biological changes at the autocrine, paracrine, and endocrine levels. Hence, it is well known that fat depots are secretory organs that control local and global metabolism (40); however, the contribution of the pCF depot to the regulation of cardiosystemic metabolism and health has remained unclear until now. Interestingly, although epicardial fat is hardly seen in rodents, the accumulation of pCF is evolutionarily conserved between species. Our study identifies ADH1 as a central regulator of $\mathrm{pCF}$ homeostasis and the $\mathrm{pCF}$ depot itself as being capable of modifying systemic metabolism. However, we cannot currently distinguish between direct $\mathrm{pCF}$-dependent regulation of systemic retinaldehyde levels and a more indirect scenario, in which it is local retinaldehyde level changes that affect the pCF secretory milieu. The fact that $A d h 1$ deletion specifically modified pCF biology suggests that this enzyme has predominant relevance in this unique fat depot. Furthermore, the impaired metabolic fitness of Adh1-KO mice subjected to cardiac exercise stress testing, and the later reversal of that impairment by removal of the fat depot, suggest that $\mathrm{pCF}$ remodeling can have functional consequences for cardiometabolic flexibility during times of stress and increased energy demands.

The identification of hormones released from adipose depots, the surgical removal of fat depots, and the genetic modification of specific types of fat have revealed that white and brown adipocytes are more than just simple sites of energy storage and wasting, as they are also capable of exerting widespread endocrine effects (refs. 41-44 and reviewed in ref. 45). Here, using surgical, genetic, and pharmacological means to modulate and remove the $\mathrm{pCF}$ depot, we demonstrate for the first time to our knowledge that this depot too is capable of effecting systemic metabolism. Whereas the metabolomics profiles of WT and $A d h 1-\mathrm{KO}$ animals were clearly different, removal of the pCF depot in both groups equalized their circulating metabolite profiles, demonstrating how the presence of this depot is capable of affecting global metabolism. Additionally, and almost surprisingly, the removal and transplant surgeries shed light on the function of this depot. Removal of the pCF depot from Adh1-KO mice improved their cardiometabolic performance and resulted in an increase in their $\mathrm{VO}_{2 \max }$, back to the levels observed in WT animals. On the contrary, transplantation of Adh1-KO pCF into WT animals resulted in reduced $\mathrm{VO}_{2 \mathrm{~m}-}$ ax and impaired cardiometabolic performance. Hence, removal of the pCF depot from Adh1-KO mice allowed these animals to reacquire some metabolic flexibility during exercise testing and, consequently, to behave more like WT animals and better rely on carbohydrate oxidation as the exercise intensity increased. Transplantation of Adh1-KO pCF into WT animals, however, had the opposite effect. The systemic retinaldehyde injections had an effect on the cardiometabolic phenotype of Adh1-KO mice that was similar to the one observed with pCF removal, while simultaneously decreasing the pCF depot size and reversing the observed whitening effect. This further supported the notation that the $\mathrm{pCF}$ depot can function as a regulator of cardiometabolic fitness in conditions of stress and that ADH1 and its product retinaldehyde are critical regulators of the depot's homeostasis.

Our previous work and the work of others have shown that global loss of ALDH1A1 prevents diet-induced iVF expansion, promotes iVF "beiging," and favors white adipocyte metabolic flexibility (4648). We observed no alterations in PCF size in ALDH1A1-deficient mice and were surprised to learn that $A d h 1$ ablation did not affect iVF mass or morphology. Instead, ablation of Adh1 modified pCF mass, mitochondrial content, and metabolic flexibility. Indeed the loss of $A d h 1$ drove maladaptive adipocyte remodeling. More specifically, pCF of the Adh1-KO mice underwent whitening, in which the distribution of unilocular adipocytes, and their size, increased. As a testament to the importance of $\mathrm{ADH} 1$ and retinaldehyde in the maintenance and regulation of this depot's biology, the pCF-specific whitening and metabolic inflexibility observed during exercise stress testing in Adh1-KO mice were rescued with systemic retinaldehyde treatment. 
The latter is extremely intriguing and hints that $\mathrm{pCF}$ remodeling is regulated through the actions of retinaldehyde. Whereas previous work has shown that the treatment of white adipocytes with retinoids can induce a change in oxidative capacity as well as in the regulation of mitochondrial genes, mitochondrial biogenesis (49), and global adiposity (50), we now observed that, through its actions on the pCF, retinaldehyde itself could induce its own set of changes. And, while it was unclear if retinaldehyde's action involved a direct antagonism of the production of secreted lipid mediators that drive obesogenic remodeling, the phenotypic changes observed in the Adh1-KO mouse - and the ability of retinaldehyde to rescue them - elucidated how its effects at the local level on pCF adipocyte and mitochondrial remodeling ultimately impacted global metabolism in conditions in which retinaldehyde homeostasis was impaired. Together, this hints at the importance of the ADH1/retinaldehyde pathway in controlling pCF depot remodeling, metabolic flexibility, and metabolism during stress. It is also important to note that chronic delivery of retinaldehyde in otherwise healthy and young animals did not perturb their homeostasis, making this molecule an attractive therapeutic agent for conditions in which pCF is pathologically remodeled, as in obesity or aging.

For over a decade, adipose tissue has been considered a main storage site for vitamin A-derived metabolites (48, 51), which function at the transcriptional level to control the expression of genes involved in fuel metabolism (refs. 52, 53 and reviewed in ref. 54) and mitochondrial biogenesis (49). A handful of alcohol and retinol dehydrogenase enzymes are capable of oxidizing retinol to retinaldehyde to facilitate the synthesis of these regulatory molecules. And, while it is clear that ADH1 is dispensable for neonatal RA biosynthesis (reviewed in refs. 55, 56), and although we also observed unchanged circulating RA levels in Adh1-KO mice, of the enzymes responsible for retinaldehyde production (ADH1, $\mathrm{ADH} 3, \mathrm{ADH} 4, \mathrm{RDH} 1$, and RDH10, reviewed in ref. 56), we only found $A d h 1$ to be expressed in our transcriptomics analysis of pCF. Thus, it appears that ADH1 has a clear and specific role in the $\mathrm{pCF}$ depot. Nonetheless, our current and previous work using the Adh1$\mathrm{KO}$ model, as well as our rescue experiments with retinaldehyde, support the notion that ADH1 functions in coordinating postnatal retinaldehyde production $(31,35,57-59)$. Indeed, we found increased conversion of retinol to retinyl esters in Adh1-KO mice, suggesting defective conversion of retinol to retinaldehyde in the absence of ADH1. This is interesting, as it also hints at cells accumulating retinaldehyde for roles that could be independent from that of RA production.

To our knowledge, the effect of retinaldehyde on coactivator translocation has not been studied, although it has been shown that retinaldehyde, as a bioactive metabolite, is capable of inducing PGC- $1 \alpha$ recruitment to the UCP1 promoter and expression of this thermogenic gene in white adipocytes $(46,60)$. PGC- $1 \alpha$ is a predominantly cytoplasmic protein, that, upon posttranslational modifications, moves into the nucleus $(61,62)$ and promotes the select activation of genetic loci associated with mitochondrial biogenesis and dynamics $(63,64)$. It is believed that PGC- $1 \alpha$ modification (i.e., acetylation and phosphorylation) is a critical mediator of the protein's activity and effect on mitochondrial homeostasis (reviewed in ref. 65), and thus posttranslational regulation of PGC-1 $\alpha$ provides a means to modulate metabolic responses. Our finding that the actions of retinaldehyde on PGC- $1 \alpha$ occur by stimulating the latter's nuclear translocation, ultimately mediating the transcription of genes involved in regulating mitochondrial dynamics, shows that retinaldehyde can regulate the function of PGC-1 $\alpha$. In our study, it was unclear whether the action of retinaldehyde on PGC-1 $\alpha$ was direct or indirect. However, outside of genome-level regulation, vitamin A metabolites containing aldehydes possess the ability to posttranslationally modify proteins by binding to positively charged and sulfur-containing amino acids through Schiff base reactions. Nonetheless, the retinaldehyde-induced effects on PGC-1 $\alpha$ occurred quickly and clearly preceded the ability of adipocytes to alter their morphology, indicating that changes at the transcriptional level, and then later at the organelle level, may drive depot remodeling. This point was further demonstrated by the ability of systemic retinaldehyde delivery to restore Opa1 expression in Adh1-KO mice and increase their mtDNA content, while also reducing their pCF mass and improving their cardiometabolic performance during exercise.

Mitochondrial function is a part of adipose tissue homeostasis, and, in obesogenic conditions, mitochondrial morphology, abundance, and content decline and are responsible for alterations in adipocyte function $(13,66-68)$. Indeed, in our work, we found that mitochondrial content, $A d h 1$, and Opa1 were all downregulated in the pCF of individuals with obesity. These findings were also mirrored in Adh1-KO mice, which phenocopy the pCF accumulation seen in individuals with obesity (69). Future work using PGC-1 $\alpha$ loss-of-function mice would represent an important corroboration of the requirement of this transcription factor for retinaldehyde-mediated regulation of the $\mathrm{pCF}$ depot.

Opportunities now exist, through the use of the Adh1-KO model or surgical pCF removal/transplant models, to further investigate the effects of this depot in conditions of obesity, aging, and heart failure. However, there are some current limits to further characterizing this depot. Specifically, there is no genetic system available for pCF-specific gene deletion or Adh1 conditional loss of function. In our study, that limitation led us to consider that the global loss of Adh1 could have alternative phenotypic effects. Despite extensive phenotypic analysis, we were unable to identify other candidates that would have allowed us to speculate about alternative causes for the observed changes in cardiometabolics and metabolomics. Moreover, by pCF surgical removal and transplantation, we demonstrated the critical importance of this fat depot in regulating systemic circulating metabolites, and, via retinaldehyde treatments or pCF removal, we showed the impact of this depot on exercise metabolic flexibility and performance. These results bring to light the importance of understanding how this depot impacts metabolic flexibility during other stressors such as heart failure-inducing insults. Our findings beg the question, "Can reversing pCF maladaptive remodeling, via surgical or retinaldehyde-based approaches, improve the metabolic flexibility and function of patients with obesity and elderly individuals?"

Altogether, our findings reveal that retinaldehyde and its upstream regulator ADH1 regulate pCF biology. The pCF depot, which we found functions to modulate cardiometabolic flexibility during exercise-induced stress, undergoes a specific whitening and accumulation with aging and obesity that was recapitulated by 
the loss of ADH1. Remodeling driven by the loss of ADH1 is accompanied by reductions in mitochondrial biogenesis and fusion that can be rescued by systemic retinaldehyde treatment. Thus, our findings highlight the therapeutic and translational potential of targeting the $\mathrm{ADH} 1 /$ retinaldehyde pathway to treat conditions of pathologic pCF expansion and dysfunction. But before this can be done, future work is required to understand the effects of retinaldehyde and $\mathrm{pCF}$ on cardiovascular disease pathogenesis.

\section{Methods}

\section{Animal generation and experimentation}

The generation of Adh1-KO mice was previously described (70). Using a gene-targeting vector, exons 7-9 from the 9-exon WT gene were deleted. Male and female WT (C57BL/6J) and Adh1-deficient mice (12-24 weeks old) were housed under a 12-hour light/12-hour dark cycle and maintained on a standard chow diet. WT mice were also subjected to a 12-week Western diet consisting of $42 \%$ of kcals from fat (TD.88137, Envigo) or to aging for 20 months. Mice had ad libitum access to food and water. All-trans retinaldehyde $(0.6 \mu \mathrm{g} / \mathrm{kg} / \mathrm{d}$, R2500, MilliporeSigma) or vehicle control was injected i.p. daily for 1 month or acutely for 6 or 24 hours. To prevent oxidation of retinaldehyde, all stock solutions were protected from light and stored under argon at $-80^{\circ} \mathrm{C}$. Experiments involving stimulation with retinaldehyde were also done in the dark, with tubes treated with argon prior to and following their use. For pCF removal and transplant studies, the depot was visualized through medial sternotomy and either simply removed (removal surgery) or removed and replaced with a pCF depot from a donor mouse (transplant surgery). See the Supplemental Methods for additional details on this surgical procedure. Mice were allowed to recover for 4 weeks before follow-up experiments.

\section{Baseline and exercise metabolic testing and chambers}

For baseline metabolic chambers, metabolic parameters were measured by indirect calorimetry. Mice were single housed in open-circuit Oxymax chambers as part of the Comprehensive Lab Animal Monitoring System (CLAMS) (Columbus Instruments). Oxymax chambers were kept on a 12-hour light/12-hour dark cycle at $23^{\circ} \mathrm{C}$. Mice had ad libitum access to food and water, and food intake was recorded. After a 24-hour acclimatization phase, oxygen consumption, carbon dioxide production, energy expenditure, and locomotion were measured. On the basis of these data, the respiratory exchange ratio (RER) $\left(\mathrm{CO}_{2} / \mathrm{O}_{2}\right), \Delta$ heat, and metabolic rate were calculated. For exercise tests, using previously described methods (28), mice were acclimated and then subjected to an endurance or graded maximal exercise test (28). In brief, for the GXT, mice were placed on the treadmill at a $0^{\circ}$ incline, and the shock grid was activated. The treadmill speed (meters), duration (minutes), and grade (degrees) were then increased until exhaustion as follows: $0 \mathrm{~m} / \mathrm{min}, 3 \mathrm{~min}, 0^{\circ}$; $6 \mathrm{~m} / \mathrm{min}, 2 \mathrm{~min}, 0^{\circ} ; 9 \mathrm{~m} / \mathrm{min}, 2 \mathrm{~min}, 5^{\circ} ; 12 \mathrm{~m} / \mathrm{min}, 2 \mathrm{~min}, 10^{\circ} ; 15 \mathrm{~m} /$ $\min , 2 \mathrm{~min}, 15^{\circ} ; 18,21,23,24 \mathrm{~m} / \mathrm{min}, 1 \mathrm{~min}, 15^{\circ}$; and $+1 \mathrm{~m} / \mathrm{min}$, each minute thereafter, $15^{\circ} \mathrm{C}$. Exhaustion (endpoint for treadmill cessation) was defined as the point at which mice maintained continuous contact with the shock grid for 5 seconds. $\mathrm{VO}_{2 \max }$ was determined by the peak oxygen consumption reached during the test when the RER was greater than 1.0. The maximum running speed was defined as the treadmill speed at which $\mathrm{VO}_{2 \max }$ was achieved.

\section{Measurement of glucose levels and body composition analysis}

Animals were fasted overnight with free access to water. BW and fasting glucose levels were determined by placing a small drop of blood from the tail onto a glucometer test strip, and levels were obtained from a OneTouch basic glucometer. Body composition analysis was performed using the EchoMRI analyzer (EchoMRI), by placing the animals in the device and performing 0.5- to 3.2-minute scans per animal to collect lean mass, fat mass, and water mass measurements in live animals without the use of anesthesia.

\section{Echocardiographic analysis, blood pressure measurement, and cardiac magnetic resonance imaging}

Echocardiographic measurements were recorded using a Vevo 2100 (VisualSonics) system and an MS-400 transducer. At baseline, the mice were anesthetized ( $1.5 \%$ isoflurane), and then ejection fraction, fractional shortening, and ventricular chamber dimensions were recorded in M-mode. Additionally, ejection fraction, fractional shortening, ventricular chamber dimensions, left ventricular mass, and heart rates were calculated using automated VevoLAB software (VisualSonics). Systolic and diastolic blood pressure measurements were recorded using the CODA Non-Invasive Blood Pressure System (Kent Scientific). After the mouse was acclimated to the blood pressure tube for 3 consecutive sessions, the O-cuff was placed close to the base of the mouse's tail. Then, the VPR cuff was placed against the O-cuff. For blood pressure recordings, 10 sequential cycles per animal were recorded and averaged. Cardiac magnetic resonance imaging (cMRI) imaging was performed using a $9.4 \mathrm{~T}$ horizontal bore imaging system (Bruker BioSpect 90/30US, Bruker BioSpin). See the Supplemental Methods for a detailed description of this procedure.

\section{Transcriptomics analysis}

RNA was isolated from the pCF and the iVF depot of WT mice using the Norgen Fatty Tissue RNA Purification Kit (Norgen Biotek) and then assessed for quality on a Bioanalyzer instrument (Agilent Technologies). Samples were then submitted to the Genomics Core, where the transcriptomic profiles were determined using the Affymetrix Mouse Transcriptome Array (version 1.0). Signal intensities were analyzed using Affymetrix Expression Console software. Background correction and quantile normalization were performed to adjust technical bias, and gene expression levels were summarized by the robust multiarray average method (71) (see the Supplemental Methods for additional details). SAS 9.4 and R statistical software programs were used for data analysis. Core Ingenuity Pathway Analysis (IPA), version 01-16 (QIAGEN), was run on significantly differentially regulated genes ( $P=0.005$ cutoff) in WT pCF and WT iVF. Expression analysis was then run on the mapped IDs on the basis of the $P$ values. Data are available in the NCBI's Gene Expression Omnibus (GEO) repository (GEO GSE162779).

\section{Metabolomics analysis}

Plasma samples for metabolomics analysis were prepared as previously described $(72,73)$. Metabolite extraction from plasma collected in K2-EDTA tubes was achieved using a mixture of isopropanol, acetonitrile, and water at a ratio of 3:3:2 v/v. Extracts were divided into 3 parts: $75 \mu \mathrm{L}$ for gas chromatography combined with timeof-flight high-resolution mass spectrometry (TOFMS), $150 \mu \mathrm{L}$ for reverse-phase liquid chromatography coupled with high-resolution 
mass spectrometry, and $150 \mu \mathrm{L}$ for hydrophilic interaction chromatography with liquid chromatography and tandem mass spectrometry, and the data were analyzed as previously described $(72,73)$. Collected raw data were manually inspected, merged, imputed, and normalized to the sample median. Additional information on metabolomics and metabolite pathway analysis is included in the Supplemental Methods.

\section{Determination of nonpolar retinoids}

Nonpolar retinoids were extracted from $200 \mu \mathrm{L}$ serum. Serum was mixed with $200 \mu \mathrm{L}$ methanol, and retinoids were extracted with a mixture containing $400 \mu \mathrm{L}$ acetone and $500 \mu \mathrm{L}$ hexanes. HPLC analysis was carried out with an Agilent 1260 Infinity Quaternary HPLC system equipped with a pump (G1312C) with an integrated degasser (G1322A), a thermostated column compartment (G1316A), an autosampler (G1329B), a diode array detector (G1315D), and online analysis software (Chemstation). The analyses were performed at $25^{\circ} \mathrm{C}$ using a normal-phase Zorbax Sil column ( $5 \mu \mathrm{m}, 4.6$ $\times 150 \mathrm{~mm}$, Agilent Technologies) protected with a guard column with the same stationary phase. Chromatographic separation was achieved by isocratic flow of $10 \%$ ethyl acetate/90\% hexanes (v/v). For quantification of molar amounts of retinoids, the HPLC was previously scaled with synthesized standard compounds (Toronto Research Chemical).

\section{Determination of RA}

For RA extraction, $300 \mu \mathrm{L}$ serum samples were homogenized in $1 \mathrm{~mL}$ PBS, pH 7.4, and then transferred into disposable glass culture tubes $(16 \mathrm{~mm} \times 150 \mathrm{~mm})$. A volume of 1-3 mL freshly prepared $0.025 \mathrm{M}$ $\mathrm{KOH}$ in ethanol was added, and the samples were vortexed. Acetonitrile $(1 \mathrm{~mL})$ was added and vortexed. A volume of $10-20 \mathrm{~mL}$ hexanes was added, and the mixture was vortexed. Phase separation was achieved by centrifugation at $500 \mathrm{~g}$ for 3 minutes. The organic top layer containing nonpolar retinoids was removed. To acidify the remaining ethanolic layer, 50-100 $\mu \mathrm{L}$ of $6 \mathrm{M}$ HCL was added, and the mixture was vortexed. Then, $10 \mathrm{~mL}$ hexanes was added, and the mixture was vortexed. Phase separation was achieved by centrifugation at $500 \mathrm{~g}$ for 3 minutes. The organic phase was removed with a glass pipette and transferred into a new glass vial. The organic solvent was evaporated under a constant stream of nitrogen. The debris was dissolved in 100-200 $\mu \mathrm{L}$ acetonitrile containing $0.1 \%$ formic acid. RA was separated on a Hypersill Gold $50 \times 2.1$ column (Thermo Fisher Scientific) by a linear gradient of $\mathrm{H}_{2} \mathrm{O} \rightarrow$ acetonitrile $(50 \%$ $\rightarrow 100 \%$ over $5 \mathrm{~min}$ followed by $100 \%$ acetonitrile for $10 \mathrm{~min}$ ) at a flow rate of $0.3 \mathrm{~mL} / \mathrm{min}$. Both solvents contained $0.1 \%$ formic acid. MS-based detection and quantification of RA were performed with an LTQ Linear Ion Trap Mass Spectrometer (Thermo Fisher Scientific) equipped with an electrospray ionization interface operated in the positive ionization mode. The MS parameters were optimized for the RA synthetic standard (Cayman Chemicals). The quantification of endogenous RA was based on the known amount of deuterated RA (dRA) (Cayman Chemicals) that served as an internal standard added to the samples prior to the analysis. RA and RRA were detected in the selected reaction monitoring (SRM) mode using the following ion transition: $301.2 \rightarrow 201.2$ and $306.3 \rightarrow 205.2$, for RA and dRA, respectively. The relationship between the SRM ion intensity peaks was used to calculate the amount of endogenous RA.

\section{Tissue staining and quantification}

Tissues were fixed in formalin, embedded in paraffin, cut into $5 \mu \mathrm{m}$ sections and stained with H\&E. Stained slides were then imaged on the EVOS Imaging System (Invitrogen, Thermo Fisher Scientific) for all experiments except the localization experiments, in which the Olympus Confocal was used. All images were imported into ImageJ (NIH) for quantification. To ensure measurement accuracy, a grid was defined to encompass the same area per tissue slide, and 8 squares within the grid were used for quantification to collect the most representative information. The number of unilocular and multilocular adipocytes was counted in each entire image from each animal. To calculate the unilocular adipocyte size, the area of each adipocyte was circled and quantified using ImageJ. To visualize muscle glycogen content, paraffin sections were stained with a periodic acid-Schiff (PAS) kit (IW3009, NovaUltra) according to the manufacturer's instructions. For immunostaining, anti-PGC-1 (1:500, NBP1-04676, Novus Biologicals) and DAPI (Life Technologies, Thermo Fisher Scientific) were used.

\section{Transmission electron microscopy}

pCF from WT and Adh1-KO mice was isolated and immediately subjected to immersion fixation using $2.5 \%$ electron microscopy (EM) grade glutaraldehyde in $0.1 \mathrm{M}$ cacodylate buffer as previously described (75). Images were captured with an FEI Tecnai G2 Biotwin transmission electron microscope (TEM) operating at $80 \mathrm{kV}$ using an AMT camera (AMT Imaging). To assess mitochondrial morphology, mitochondrial size, and aspect ratio (ratio of length/width) were quantified using ImageJ.

\section{Western blotting and triglyceride assays}

Protein extracts from pCF tissue were obtained in extraction buffer (10 mM HEPES [pH 7.6], $60 \mathrm{mM} \mathrm{KCl,} 1$ mM EDTA, 0.1\% nonidet P-40, 1 mM DTT). For fractionation of nuclei and cytoplasm, nuclei were pelleted $\left(200 \mathrm{~g}, 4^{\circ} \mathrm{C}, 5 \mathrm{~min}\right)$, and cytoplasmic supernatants were transferred into fresh tubes. Nuclei were then washed with cytoplasmic extraction buffer without Nonidet P-40 (Sigma-Aldrich) and again pelleted $\left(200 \mathrm{~g}, 4^{\circ} \mathrm{C}, 5 \mathrm{~min}\right)$. Nuclear extraction buffer $(20 \mathrm{mM}$ Tris [pH 8.0], $400 \mathrm{mM} \mathrm{NaCl}, 1.5 \mathrm{mM} \mathrm{MgCl}, 0.2 \mathrm{mM}$ EDTA, $0.5 \mathrm{mM}$, $25 \%$ glycerol) was then added. Standard Western blotting analysis was performed using the following primary antibodies: PGC-1 $\alpha$ (1:2000, NBP1-04676, Novus Biologicals); histone 3 (1:2000, NBP1-30141, Novus Biologicals); Oxphos cocktail (1:5000, ab110413, Abcam); $\beta$-actin (1:5000, 60008-1-Ig, Proteintech); UCP1 (1:500, NBP2-20796, Novus Biologicals); and GAPDH (1:10,000, 10R-G109a, Fitzgerald Industries). Secondary antibody incubations were done at room temperature for 90 minutes using HRP-conjugated antibodies $(1: 10,000)$ and then imaged using a ChemiDoc system (Bio-Rad). Triglyceride assays were performed using Wako Diagnostic's triglyceride kit according to the manufacturer's instructions (see the Supplemental Methods for an extended description of this procedure).

\section{Cell cultures}

ATCC NIH-3T3 fibroblasts were transiently transfected in triplicate (Lipofectamine 3000, Thermo Fisher Scientific) at $80 \%$ confluence with full-length human PPAR $\gamma$ subcloned into a pcDNA vector and with an siRNA control or siRNA against $A d h 1$. For experiments using American Type Culture Collection (ATCC) NIH 3T3-L1 preadipocytes, the transfections were combined with adipogenic media (10\% FBS, 10 
$\mathrm{mg} / \mathrm{mL}$ insulin, $1 \mu \mathrm{m}$ dexamethasone, $0.5 \mathrm{mM}$ 3-isobutyl-1-methylxanthine (IBMX). Cells were assayed 48 hours after transfection. All cell culture reagents were obtained from MilliporeSigma, with the exception of insulin (from Invitrogen, Thermo Fisher Scientific) and IBMX media (from Gibco, Thermo Fisher Scientific).

\section{mRNA and mtDNA expression analysis}

RNA was extracted using TRIzol (Thermo Fisher Scientific) as previously described (76) and then reverse-transcribed using the High Capacity cDNA Reverse Transcription kit (Applied Biosystems). Selected gene expression differences were analyzed by real-time quantitative PCR (qPCR) using SsoAdvanced SYBR Green Supermix (Bio-Rad) in a 96-well format. Quantified mRNA levels were normalized to the housekeeping gene, and expression is presented relative to control levels. For mtDNA quantification, DNA was isolated from fresh tissue or from paraffin-embedded blocks using proteinase $\mathrm{K}$ digests followed by phenol-isopropanol extractions. Isolated DNA was resuspended in water and then diluted to $4 \mathrm{ng} / \mu \mathrm{L}$, and $\mathrm{qPCR}$ reactions were set up as described above. The primers used are listed in the Supplemental Methods.

\section{Statistics}

All results are presented as the mean \pm SEM. Statistical analysis was performed using the Student's 2-tailed $t$ test for normally distributed data. $P$ values of 0.05 or less were considered statistically significant. For groups of 3, a 1-way ANOVA followed by Tukey's honestly significant difference (HSD) multiple-comparison test was performed, with statistical significance set at $\alpha=0.05$. For groups of 2 genotypes and 2 conditions, a 2-way ANOVA followed by Tukey's HSD multiple-comparison test was performed, with statistical significance set at $\alpha=0.05$. Data analysis was performed using GraphPad Prism 8 (GraphPad Software).

\section{Study approval}

The use of animals in this study was approved by the IACUC of The Ohio State University. The studies using human tissue were performed under the guidelines of the Declaration of Helsinki, with approval of the IRBs of The Ohio State University, as previously described (77). Samples of pCF tissue were obtained from human donors in collaboration with the Lifeline of Ohio Organ Procurement program. The hearts used in this study were not suitable for transplantation, and their donors died from causes other than heart failure. pCF was removed from the donors and immediately flash-frozen in liquid nitrogen and stored at $-80^{\circ} \mathrm{C}$ until use.

\section{Author contributions}

JMP, OZ, and FA conceived the project. JMP and FA wrote and revised the manuscript. JMP performed most of the in vivo and ex vivo studies involving mtDNA and mRNA expression analysis, Western blotting, transmission electron microscopy, immunofluorescence staining and imaging, glucose testing, exercise testing and rescue injections and experiments. XX and JMP performed pCF removal studies. JZL performed Western diet feeding and glucose testing. LY performed transcriptomics analysis. SR and JVL performed retinoid measurements. JMP, MAK, VT, and VB performed metabolomics sample analysis and data interpretation. SM, MP, and JMP performed baseline metabolic experiments. LED performed echocardiography. GD generated the Adh1-KO model. PMLJ provided samples for human $\mathrm{pCF}$ studies. JMP, OZ, FA, AB, JZL, LY, MP, SM, and JVL edited the manuscript. FA and $\mathrm{OZ}$ supervised the entire project.

\section{Acknowledgments}

We wish to thank Jan Czekajewski, Ron Campbell, and Chris Adams at Columbus Instruments for providing access to their state-of-the-art equipment for our metabolic treadmill testing experiments. We thank the Lifeline of Ohio Organ Procurement service for their collaboration in obtaining human cardiac tissue. We are grateful to J. White for helpful discussions and review of the manuscript. We are thankful to S. Warner, A. Flechtner, P. Fadda, and S. Mikula at the core facilities of The Ohio State University (CCSG, P30CA016058). Body composition analysis and echocardiography were performed at the Small Animal Imaging Core of The Ohio State University. This work was supported by the National Institute of General Medical Sciences, NIH under (T32GM068412, to JMP) and the National Institute of Arthritis and Musculoskeletal and Skin Diseases, NIH (F31AR073638, to JMP). This work was also supported by grants from the NIH (HL121284 and HL136951, to FA) and the American Heart Association (AHA) (17IRG33460198, to FA).

Address correspondence to: Ouliana Ziouzenkova, Department of Human Nutrition, Campbell Hall, The Ohio State University, 331A Campbell Hall, Columbus, OH, 43210, USA. Phone: 614.292.5034; Email: Ziouzenkova.1@osu.edu. Or to: Federica Accornero, Department of Physiology and Cell Biology, Dorothy M. Davis Heart and Lung Research Institute, The Ohio State University, $473 \mathrm{~W}$ 12th Ave, Columbus, OH 43210, USA. Phone: 614.366.1058; Email: federica.accornero@osumc.edu.
1. Choe SS, et al. Adipose tissue remodeling: its role in energy metabolism and metabolic disorders. Front Endocrinol (Lausanne). 2016;7:30.

2. Zwick RK, et al. Anatomical, physiological, and functional diversity of adipose tissue. Cell Metab. 2018;27(1):68-83.

3. $\mathrm{Cao} \mathrm{H}$, et al. Identification of a lipokine, a lipid hormone linking adipose tissue to systemic metabolism. Cell. 2008;134(6):933-944.

4. Lee MJ, et al. Adipose tissue remodeling in pathophysiology of obesity. Curr Opin Clin Nutr Metab Care. 2010;13(4):371-376.

5. Rondinone CM. Adipocyte-derived hor- mones, cytokines, and mediators. Endocrine. 2006;29(1):81-90.

6. Goodpaster BH, Sparks LM. Metabolic flexibility in health and disease. Cell Metab. 2017;25(5):1027-1036.

7. Sparks LM, et al. Relation of adipose tissue to metabolic flexibility. Diabetes Res Clin Pract. 2009;83(1):32-43.

8. Smith RL, et al. Metabolic flexibility as an adaptation to energy resources and requirements in health and disease. Endocr Rev. 2018;39(4):489-517.

9. Choo HJ, et al. Mitochondria are impaired in the adipocytes of type 2 diabetic mice. Diabetologia. 2006;49(4):784-791.

10. Wilson-Fritch L, et al. Mitochondrial biogenesis and remodeling during adipogenesis and in response to the insulin sensitizer rosiglitazone. Mol Cell Biol. 2003;23(3):1085-1094.

11. Wilson-Fritch L, et al. Mitochondrial remodeling in adipose tissue associated with obesity and treatment with rosiglitazone. J Clin Invest. 2004;114(9):1281-1289.

12. Muoio DM. Metabolic inflexibility: when mitochondrial indecision leads to metabolic gridlock. Cell. 2014;159(6):1253-1262. 
13. Kusminski CM, Scherer PE. Mitochondrial dysfunction in white adipose tissue. Trends Endocrinol Metab. 2012;23(9):435-443.

14. Iacobellis $G$, et al. Epicardial adipose tissue: anatomic, biomolecular and clinical relationships with the heart. Nat Clin Pract Cardiovasc Med. 2005;2(10):536-543.

15. Iacobellis G, Willens HJ. Echocardiographic epicardial fat: a review of research and clinical applications. J Am Soc Echocardiogr. 2009;22(12):1311-1319.

16. Ouwens DM, et al. The role of epicardial and perivascular adipose tissue in the pathophysiology of cardiovascular disease. JCell Mol Med. 2010;14(9):2223-2234.

17. Sacks HS, Fain JN. Human epicardial adipose tissue: a review. Am Heart J. 2007;153(6):907-917.

18. Rabkin SW. Epicardial fat: properties, function and relationship to obesity. Obes Rev. 2007;8(3):253-261.

19. Tchkonia T, et al. Mechanisms and metabolic implications of regional differences among fat depots. Cell Metab. 2013;17(5):644-656.

20. Hutley L, Prins JB. Fat as an endocrine organ: relationship to the metabolic syndrome. Am J Med Sci. 2005;330(6):280-289.

21. Ottaviani $\mathrm{E}$, et al. The evolution of the adipose tissue: a neglected enigma. Gen Comp Endocrinol. 2011;174(1):1-4

22. Yamaguchi $Y$, et al. Adipogenesis and epicardial adipose tissue: a novel fate of the epicardium induced by mesenchymal transformation and PPAR $\gamma$ activation. Proc Natl Acad Sci U S A. 2015;112(7):2070-2075.

23. Iacobellis $G$, Malavazos AE. Pericardial adipose tissue, atherosclerosis, and cardiovascular disease risk factors: the Jackson Heart Study: comment on Liu, et al. Diabetes Care. 2010;33(9):e127.

24. Ansaldo AM, et al. Epicardial adipose tissue and cardiovascular diseases. Int J Cardiol. 2019;278:254-260.

25. Mahabadi AA, et al. Association of epicardial fat with cardiovascular risk factors and incident myocardial infarction in the general population: the Heinz Nixdorf Recall Study. J Am Coll Cardiol. 2013;61(13):1388-1395.

26. Malavazos AE, et al. Relation of echocardiographic epicardial fat thickness and myocardial fat. Am J Cardiol. 2010;105(12):1831-1835.

27. Wang CP, et al. Increased epicardial adipose tissue (EAT) volume in type 2 diabetes mellitus and association with metabolic syndrome and severity of coronary atherosclerosis. Clin Endocrinol (Oxf). 2009;70(6):876-882.

28. Petrosino JM, et al. Graded maximal exercise testing to assess mouse cardio-metabolic phenotypes. PLoS One. 2016;11(2):e0148010.

29. Heilbronn LK, et al. Markers of mitochondrial biogenesis and metabolism are lower in overweight and obese insulin-resistant subjects. JClin Endocrinol Metab. 2007;92(4):1467-1473.

30. Yin X, et al. Adipocyte mitochondrial function is reduced in human obesity independent of fat cell size. J Clin Endocrinol Metab. 2014;99(2):E209-E216.

31. Molotkov A, Duester G. Genetic evidence that retinaldehyde dehydrogenase Raldh1 (Aldh1a1) functions downstream of alcohol dehydrogenase Adh1 in metabolism of retinol to retinoic acid. J Biol Chem. 2003;278(38):36085-36090.

32. DiSilvestro D, et al. Enzymatic intracrine regulation of white adipose tissue. Horm Mol Biol Clin Investig. 2014;19(1):39-55.

33. Duester G, et al. Cytosolic retinoid dehydrogenases govern ubiquitous metabolism of retinol to retinaldehyde followed by tissue-specific metabolism to retinoic acid. Chem Biol Interact. 2003;143-144:201-210.

34. Kumar S, et al. Alcohol and aldehyde dehydrogenases: retinoid metabolic effects in mouse knockout models. Biochim Biophys Acta. 2012;1821(1):198-205.

35. Yasmeen R, et al. The contribution of vitamin A to autocrine regulation of fat depots. Biochim Biophys Acta. 2012;1821(1):190-197.

36. Ding J, et al. The association of pericardial fat with incident coronary heart disease: the Multi-Ethnic Study of Atherosclerosis (MESA). Am JClin Nutr. 2009;90(3):499-504.

37. Iozzo P. Myocardial, perivascular, and epicardial fat. Diabetes Care. 2011;34 Suppl 2:S371-S379.

38. Costa RM, et al. Perivascular adipose tissue as a relevant fat depot for cardiovascular risk in obesity. Front Physiol. 2018;9:253.

39. Houben AJ, et al. Perivascular fat and the microcirculation: relevance to insulin resistance, diabetes, and cardiovascular disease. Curr Cardiovasc Risk Rep. 2012;6(1):80-90.

40. Hildebrand S, et al. PVAT and its relation to brown, beige, and white adipose tissue in development and function. Front Physiol. 2018;9:70.

41. Ahima RS, Flier JS. Adipose tissue as an endocrine organ. Trends Endocrinol Metab. 2000;11(8):327-332.

42. Enerback S, et al. Mice lacking mitochondrial uncoupling protein are cold-sensitive but not obese. Nature. 1997;387(6628):90-94.

43. Gabriely I, et al. Removal of visceral fat prevents insulin resistance and glucose intolerance of aging: an adipokine-mediated process? Diabetes. 2002;51(10):2951-2958.

44. Cox-York K, et al. Lower body adipose tissue removal decreases glucose tolerance and insulin sensitivity in mice with exposure to high fat diet. Adipocyte. 2015;4(1):32-43.

45. Fruhbeck $\mathrm{G}$, et al. The adipocyte: a model for integration of endocrine and metabolic signaling in energy metabolism regulation. Am J Physiol Endocrinol Metab. 2001;280(6):E827-E847.

46. Kiefer FW, et al. Retinaldehyde dehydrogenase 1 regulates a thermogenic program in white adipose tissue. Nat Med. 2012;18(6):918-925.

47. Yasmeen R, et al. Autocrine function of aldehyde dehydrogenase 1 as a determinant of diet- and sex-specific differences in visceral adiposity. Diabetes. 2013;62(1):124-136.

48. Ziouzenkova O, et al. Retinaldehyde represses adipogenesis and diet-induced obesity. Nat Med. 2007;13(6):695-702.

49. Tourniaire F, et al. All-trans retinoic acid induces oxidative phosphorylation and mitochondria biogenesis in adipocytes. J Lipid Res. 2015;56(6):1100-1109.

50. Mercader J, et al. Remodeling of white adipose tissue after retinoic acid administration in mice.
Endocrinology. 2006;147(11):5325-5332.

51. Tourniaire F, et al. beta-Carotene conversion products and their effects on adipose tissue. Genes Nutr. 2009;4(3):179-187.

52. Kane MA, et al. CrbpI modulates glucose homeostasis and pancreas 9-cis-retinoic acid concentrations. Mol Cell Biol. 2011;31(16):3277-3285.

53. Schwarz EJ, et al. Retinoic acid blocks adipogenesis by inhibiting C/EBPbeta-mediated transcription. Mol Cell Biol. 1997;17(3):1552-1561.

54. Ziouzenkova O, Plutzky J. Retinoid metabolism and nuclear receptor responses: New insights into coordinated regulation of the PPAR-RXR complex. FEBS Lett. 2008;582(1):32-38.

55. Kedishvili NY. Enzymology of retinoic acid biosynthesis and degradation. J Lipid Res. 2013;54(7):1744-1760.

56. Duester G. Retinoic acid synthesis and signaling during early organogenesis. Cell. 2008;134(6):921-931.

57. Yang K, et al. Aldehyde dehydrogenase $1 \mathrm{a} 1$ regulates energy metabolism in adipocytes from different species. Xenotransplantation. 2017;24(5):e12318.

58. Petrosino JM, et al. Aldehyde dehydrogenase 1A1: friend or foe to female metabolism? Nutrients. 2014;6(3):950-973.

59. Reichert B, et al. Concerted action of aldehyde dehydrogenases influences depot-specific fat formation. Mol Endocrinol. 2011;25(5):799-809.

60. Puigserver $\mathrm{P}$, et al. A cold-inducible coactivator of nuclear receptors linked to adaptive thermogenesis. Cell. 1998;92(6):829-839.

61. Jager S, et al. AMP-activated protein kinase (AMPK) action in skeletal muscle via direct phosphorylation of PGC-1alpha. Proc Natl Acad Sci US A. 2007;104(29):12017-12022.

62. Lagouge M, et al. Resveratrol improves mitochondrial function and protects against metabolic disease by activating SIRT1 and PGC-1alpha. Cell. 2006;127(6):1109-1122.

63. Cartoni R, et al. Mitofusins $1 / 2$ and ERRalpha expression are increased in human skeletal muscle after physical exercise. J Physiol. 2005;567(Pt 1):349-358.

64. Greene NP, et al. Mitochondrial quality control, promoted by PGC-1 $\alpha$, is dysregulated by Western diet-induced obesity and partially restored by moderate physical activity in mice. Physiol Rep. 2015;3(7):e12470

65. Jeninga EH, et al. Reversible acetylation of PGC-1: connecting energy sensors and effectors to guarantee metabolic flexibility. Oncogene. 2010;29(33):4617-4624.

66. Flachs $P$, et al. Stimulation of mitochondrial oxidative capacity in white fat independent of UCP1: a key to lean phenotype. Biochim Biophys Acta. 2013;1831(5):986-1003.

67. Medina-Gomez G. Mitochondria and endocrine function of adipose tissue. Best Pract Res Clin Endocrinol Metab. 2012;26(6):791-804.

68. Wang Y, et al. Translocase of inner mitochondrial membrane 44 alters the mitochondrial fusion and fission dynamics and protects from type 2 diabetes. Metabolism. 2015;64(6):677-688.

69. Aldiss P, et al. 'Browning' the cardiac and peri-vascular adipose tissues to modulate cardiovascular risk. Int J Cardiol. 2017;228:265-274.

70. Deltour L, et al. Metabolic deficiencies in 
alcohol dehydrogenase Adh1, Adh3, and Adh4 null mutant mice. Overlapping roles of Adh1 and Adh 4 in ethanol clearance and metabolism of retinol to retinoic acid. J Biol Chem. 1999;274(24):16796-16801.

71. Irizarry RA, et al. Exploration, normalization, and summaries of high density oligonucleotide array probe level data. Biostatistics. 2003;4(2):249-264.

72. Baskin AS, et al. Regulation of human adipose tissue activation, gallbladder size, and bile acid metabolism by a $\beta 3$-adrenergic receptor agonist. Diabetes. 2018;67(10):2113-2125.

73. Drolet J, et al. Integrated metabolomics assessment of human dried blood spots and urine strips. Metabolites. 2017;7(3):35.

74. Tolstikov V, et al. Metabolomics analysis of metabolic effects of nicotinamide phosphoribosyltransferase (NAMPT) inhibition on human cancer cells. PLoS One. 2014;9(12):e114019.
75. Ackermann MA, et al. TGF- $\beta 1$ affects cell-cell adhesion in the heart in an NCAM1-dependent mechanism. J Mol Cell Cardiol. 2017;112:49-57.

76. Accornero F, et al. BEX1 is an RNA-dependent mediator of cardiomyopathy. Nat Commun. 2017;8(1):1875.

77. Chung JH, et al. Etiology-dependent impairment of relaxation kinetics in right ventricular endstage failing human myocardium. J Mol Cell Cardiol. 2018;121:81-93. 